\title{
Dispersion Reducing Methods for Edge Discretizations of the Electric Vector Wave Equation
}

\author{
V. A. Bokil ${ }^{\mathrm{a}, 1}$, N. L. Gibson ${ }^{\mathrm{a}, 2}$, V. Gyrya ${ }^{\mathrm{b}, 3}$, D. A. McGregor ${ }^{\mathrm{a}, 4, *}$ \\ ${ }^{a}$ Department of Mathematics, Oregon State University, Corvallis, OR, 97330 \\ ${ }^{b}$ T5: Applied Mathematics and Plasma Physics, Los Alamos National Laboratory, Los Alamos, NM 87545
}

\begin{abstract}
We present a novel strategy for minimizing the numerical dispersion error in edge discretizations of the time-domain electric vector wave equation on square meshes based on the mimetic finite difference (MFD) method. We compare this strategy, called $M$-adaptation, to two other discretizations, also based on square meshes. One is the lowest order Nédélec edge element discretization. The other is a modified quadrature approach ( $G Y$-adaptation) proposed by Guddati and Yue for the acoustic wave equation in two dimensions. All three discrete methods use the same edge-based degrees of freedom, while the temporal discretization is performed using the standard explicit Leapfrog scheme. To obtain efficient and explicit time stepping methods, the three schemes are further mass lumped. We perform a dispersion and stability analysis for the presented schemes and compare all three methods in terms of their stability regions and phase error. Our results indicate that the method produced by GY-adaptation and the Nédélec method are both second order accurate for numerical dispersion, but differ in the order of their numerical anisotropy (fourth order, versus second order, respectively). The result of M-adaptation is a discretization that is fourth order accurate for numerical dispersion as well as numerical anisotropy. Numerical simulations are provided that illustrate the theoretical results.
\end{abstract}

Keywords: Maxwell's equations, vector wave equation, Nédélec edge elements, M-adaptation, dispersion, anisotropy.

2000 MSC: 65M60, 78M10

\section{Introduction}

We consider numerical solutions of the two-dimensional time-domain electric vector wave equation on square meshes using the classical Leapfrog time discretization. Although we restrict ourselves to time domain formulations, the ideas presented here can also be applied to frequency domain formulations. The design and characterization of many pulsed microwave and millimeter wave devices requires the time domain analysis of electromagnetic (EM)

\footnotetext{
${ }^{*}$ Corresponding author

${ }^{1}$ email: bokilv@math.oregonstate.edu

${ }^{2}$ email: gibsonn@math.oregonstate.edu

${ }^{3}$ email: vitaliy_gyrya@lanl.gov

${ }^{4}$ email: mcgregod@math.oregonstate.edu
} 
wave propagation $[2,14]$. Popular numerical methods for simulating EM wave propagation are generally either based on the Finite Element (FE) or on the Finite Difference (FD) methods. The curl-conforming finite element discretizations (edge elements), first developed by Nédélec [25, 26], are very popular for the spatial discretization of the first order Maxwell equations or the second order vector wave equation. The popularity of edge discretizations is due to their ability to eliminate the non-physical (spurious) modes that are present in discretizations of the vector wave equation based on nodal finite elements $[12,22,23,24,30]$. Edge discretizations also allow for easy implementation of boundary and material interface conditions by automatically ensuring the continuity of the tangential components of the vector fields [16, 20, 32].

One of the issues with FE discretizations for wave equations in general, and the electric wave equation in particular, is that a non-diagonal mass matrix has to be inverted to solve a linear system during every time step of the solution of the discrete system. This is highly inefficient for a problem where the number of time steps is typically on the order of the number of spatial steps (due to a Courant-type stability condition). A popular remedy has been an approximation of the mass matrix $M$ with a diagonal matrix $D$ obtained by lumping all entries of the mass matrix to the diagonal. Hence, the name for the procedure - mass lumping. Mass-lumped methods maintain the same order of the dispersion error as their non-lumped counterparts, however they often suffer from larger constants in their dispersion errors [21].

One of the most popular FD methods for the numerical discretization of Maxwell's equations is the Yee scheme [33] which has been shown to be equivalent to a mass-lumped version of the lowest order Nédélec FE method on a uniform cubic mesh [19]. An extensive overview of numerical methods for wave equation in the time domain can be found in [5]. This includes high order finite difference, high order finite element methods, and numerical dispersion analysis for the acoustics, linear elasticity, and Maxwell's equations. There are versions of FD methods for unstructured grids [31], but these methods are easiest to implement on structured grids. In this paper we will focus on discretizations on square meshes that are optimized for their numerical dispersion error.

Bokil and Gibson [3] considered a number of FD discretizations of Maxwell's equations for which they demonstrated reduction of numerical dispersion when the spatial discretization order was increased from second to fourth while maintaining second order for the temporal discretization. Increasing the spatial discretization beyond fourth order did not produce any significant reduction in the dispersion error. Smith, et al. [28] were able to eliminate fourth order dispersion error by using a modified fourth-order FD method in space and time. The modified stencil of the FD method was obtained as a weighted average of various rotated stencils.

The problem of constructing methods with optimal numerical dispersion properties has been considered in the case of acoustics, where similar results were obtained using FD and FE-type discretizations. In particular, Jo et al. [13] and Stekl and Pratt [29] derived an optimized 9-point FD method in two space dimensions for an acoustic wave equation in the frequency domain. Their idea was to consider a weighted average of the standard and "diagonal" FD methods and optimize the resulting scheme for the weights. Sescu et al. [27] extended this idea to three space dimensions.

One can approach the reduction of dispersion with time domain finite element methods 
(TDFEM) by employing higher order discretizations. However, a well known drawback of this approach is the necessity of inverting the mass matrix at every time step. Fisher et al. in [7] addressed this issue by employing a generalized mass lumping technique. Their approach was to increase the computational efficiency of high order Nédélec Elements by choosing GaussLobatto quadrature points and using these quadratures to resolve the integrals of the mass and stiffness matrices. On orthogonal Cartesian meshes their method produces a diagonal mass matrix and on more general geometries the sparsity of the mass matrix is greatly reduced if non-diagonal. As is typical of Nédélec elements, they recover a dispersion error of order $2 p$ where $p$ is the polynomial degree. This approach is philosophically different than our approach: while they attempt to make an accurate method more efficient, we will make an efficient method more accurate. Our optimization technique produces a method which has a formal $L^{2}$ error of order two and dispersion error of order four producing higher quality solutions than those produced by the lowest order Nédélec elements. A natural extension of our work is the development of both higher order discretizations using M-adaptation and an extension to hexahedral meshes.

Another technique based on the FE framework is closer to our method, see e.g., [8, 15, 34]. Instead of using the typical lumping, i.e., by approximating the mass matrix $M$ with a diagonal matrix $D$, an approximation of the mass matrix $M \approx D M^{-1} D$ is used. This approach makes approximation of $M^{-1} \approx D^{-1} M D^{-1}$ simple, as it involves inverting a diagonal matrix $D$. Also, in this approach the mass matrix $M$ is retained in the scheme, which becomes important in the optimization step. The optimization step is similar in spirit to utilizing weighted combinations of standard and rotated stencils in the FD approach.

In $[8,34]$ Guddati and Yue considered approximating the mass and stiffness matrices using quadrature rules, instead of employing exact integration. The use of quadrature rules allowed the characterization of each matrix by one quadrature parameter. The values of the parameters were selected to minimize the numerical dispersion error. We refer to this approach as $G Y$-adaptation.

In $[9,10]$ Gyrya and Lipnikov considered a generalization of FE and staggered FD discretizations to general polygonal meshes, called Mimetic Finite Difference (MFD) methods, (see $[4,17]$ and references therein). The MFD approach produces a large parametrized family of schemes with equivalent properties, such as stencil size and base convergence rate among others. The number of parameters characterizing the scheme grows rapidly with the dimension, the number of vertices in a polygonal element, and the order of the discretization. For the acoustics equation in $2 \mathrm{D}$ on rectangular meshes the stiffness matrix was characterized by one parameter and the mass matrix was characterized by three parameters (two on square meshes, due to symmetry). The process of selecting an optimal member for a selected optimization criteria (in this case, minimization of numerical dispersion error) within the MFD family was called, M-adaptation. In principle, other optimization criteria could be selected, see e.g., [11]. On rectangular meshes the optimal member of the MFD family was shown to have fourth-order (vs. second-order) numerical dispersion and fourth-order (vs. secondorder) numerical anisotropy. The Guddati and Yue approach [8,34] did not produce any reduction in the numerical dispersion or numerical anisotropy on non-square meshes. On square meshes, using M-adaptation, the numerical anisotropy was further reduced to be of sixth-order (vs. second-order).

In this paper, following the ideas developed for nodal discretizations for the acoustics 
equation in $[8,9,10,34]$, we consider edge discretizations of the vector wave equation and apply the techniques of GY- and M-adaptation to produce novel schemes that are optimized for their numerical dispersion properties. In particular we will utilize the non-standard lumping approach for the mass matrix that was considered in [8, 15, 34] and described above. The derivation of the dispersion relation will be a generalization of the approach used in [10]. In the case of the vector wave equation, the dispersion relation takes a vectormatrix form, unlike the scalar form for the acoustics equation, which makes the analysis of numerical dispersion of discretizations of the vector wave equation more challenging. Also, unlike the case of the nodal discretization for the acoustic equation, the stiffness matrix is defined uniquely (i.e., does not depend on quadrature parameters); thus all parameters are located in the mass matrix.

The paper is organized as follows. In Section 2 we present the initial value problem for the vector wave equation and its variational formulation. In Section 3 we present a common framework for the three discretizations to be considered here: Nédélec, GY family of methods and MFD family of methods. All discretizations are first presented on rectangular meshes, but later most of the results will be obtained for square meshes. The common framework will allow us to use similar stability and dispersion analysis for the three methods. We present the dispersion relation for the continuous PDE formulation of the vector wave equation and derive the dispersion relation for the three numerical discretizations in Section 4. In Section 5 we produce the dispersion relation and calculate a numerical phase error for each of the numerical discretizations. For the GY family and the MFD family we identify the "optimal" members that have minimum numerical dispersion and anisotropy among all members in their respective families. In Section 6 we perform von Neumann stability analysis on all three methods and we identify Courant-type stability conditions. Of the three methods analyzed we find that GY-adaptation is the least stable and the Nédélec method is the most stable. Finally, in Section 7 we present computational tests that illustrate the reduction of the numerical dispersion for each of the numerical discretizations, as predicted from the dispersion relation, and in Section 8 we present some concluding remarks.

\section{PDE Formulation}

We present Maxwell's equations first in the classical differential formulation in Section 2.1 and then reformulated in the weak form in Section 2.2. The latter formulation forms the foundation for finite element discretizations as well as other discretizations considered in this paper.

\subsection{Classical formulation}

Let $\Omega \subset \mathbb{R}^{2}$ be a domain with Lipschitz boundary $\partial \Omega=\Gamma$. Consider the two dimensional transverse electric (TE) formulation of Maxwell's equations in a linear medium, given by the 
following four equations

$$
\begin{aligned}
& \frac{\partial H}{\partial t}=-\frac{1}{\mu} \operatorname{curl} \mathbf{E}, \text { on }(0, T] \times \Omega, \\
& \frac{\partial \mathbf{E}}{\partial t}=\frac{1}{\epsilon} \operatorname{curl} H-\mathbf{J}, \text { on }(0, T] \times \Omega, \\
& \nabla \cdot \epsilon \mathbf{E}=0, \text { on }(0, T] \times \Omega, \\
& \mathbf{E}(x, 0)=\mathbf{E}_{0}, H(x, 0)=H_{0}, \text { on } \Omega,
\end{aligned}
$$

along with appropriate boundary conditions. In the system (2.1), E is the electric (vector) field, while $H$ is the (scalar) magnetic field. The variable $\mathbf{J}$ is the current density and the parameters $\epsilon$, and $\mu$, are the permittivity, and permeability of the linear medium, respectively. For a vector field $\mathbf{f}=\left(\mathbf{f}_{x}, \mathbf{f}_{y}\right)^{T}$ and for a scalar field $f$ we define the scalar (curl) and vector (curl) curl operators as follows, [18],

$$
\operatorname{curl}(\mathbf{f}):=\frac{\partial}{\partial x} \mathbf{f}_{y}-\frac{\partial}{\partial y} \mathbf{f}_{x} \quad \operatorname{curl}(f):=\left(\frac{\partial}{\partial y} f, \frac{\partial}{\partial x} f\right)^{T} .
$$

All the fields in the system (2.1) are functions of position $\mathbf{x}=(x, y)^{T}$ and time $t \in[0, T]$. Assuming sufficient differentiability of the solution and the data of system (2.1), taking the time derivative of equation $(2.1 \mathrm{~b})$ we can eliminate the magnetic field $H$ to obtain a two dimensional vector wave equation in the electric field only. This yields a second order formulation of the Maxwell system given as the 2D Electric Vector Wave Equation

$$
\begin{aligned}
& \frac{\partial^{2}}{\partial t^{2}} \mathbf{E}+c^{2} \text { curl curl } \mathbf{E}=-\frac{\partial}{\partial t} \mathbf{J}, \\
& \nabla \cdot \epsilon \mathbf{E}=0
\end{aligned}
$$

where $c=1 / \sqrt{\epsilon \mu}$ is the speed of light in the medium.

Remark. The above formulation is very similar to the 2D Magnetic Vector Wave Equation. This formulation is derived by assuming that $\mathbf{H}$ has only $x$ and $y$ components while $E$ has only $z$ components. The primary difference of the two methods is that $\frac{\partial}{\partial t} \mathbf{J}$ must be replaced with curl $J$. Therefore the following work applies as easily to transverse magnetic problems as to transverse electric problems.

\subsection{Variational formulation}

To construct our numerical methods we start with a variational formulation of the two dimensional vector wave equation (2.2). We assume periodic boundary conditions and consider the following Sobolev spaces

$$
\begin{aligned}
\mathbf{L}^{2}(\Omega) & =\left[L^{2}(\Omega)\right]^{2} \\
\mathbf{H}(\operatorname{curl}, \Omega) & =\left\{\mathbf{v} \in \mathbf{L}^{2}(\Omega): \operatorname{curl} \mathbf{v} \in L^{2}(\Omega)\right\} .
\end{aligned}
$$

Multiplying (2.2a) by a test function $\phi \in \mathbf{H}(\operatorname{curl}, \Omega)$ and integrating over $\Omega$ we obtain the variational formulation: 
Find $\mathbf{E} \in C^{2}([0, T] ; \mathbf{H}(\operatorname{curl}, \Omega))$, such that for all $\boldsymbol{\phi} \in \mathbf{H}(\operatorname{curl}, \Omega)$

$$
\int_{\Omega} \frac{\partial^{2}}{\partial t^{2}} \mathbf{E} \cdot \boldsymbol{\phi} d \Omega+c^{2} \int_{\Omega}(\operatorname{curl} \mathbf{E}) \cdot(\operatorname{curl} \phi) d \Omega=-\int_{\Omega} \frac{\partial}{\partial t} \mathbf{J} \cdot \boldsymbol{\phi} d \Omega,
$$

subject to initial conditions $\mathbf{E}(0)=\mathbf{E}_{0}$ and $\frac{\partial}{\partial t} \mathbf{E}(0)=\frac{1}{\epsilon} \operatorname{curl} H_{0}$.

Remark. For weak solutions to be physically meaningful it is important that given initial conditions which satisfy the divergence constraint, that for all time the weak solutions satisfy the divergence constraint.

\section{Numerical Discretizations}

In this section we will consider several discretizations of $(2.3)$ in $2 \mathrm{D}$ where the discrete degrees of freedom (d.o.f.) representing the electric field will be defined in a uniform way. The only difference between the discretizations is the approximation of the bilinear forms. We represent the scalar magnetic field $H$ on a polygonal element $P$ using a single value which we take to be an average of $H$ over the element. We represent the vector electric field $\mathbf{E}$ by the average values of the tangent component, of $\mathbf{E}$ on every edge, see Figure 1. We refer to our degrees of freedom (d.o.f.) as $E_{j}$,

$$
E_{j}=\frac{1}{\left|e_{j}\right|} \int_{e_{j}} \boldsymbol{\tau}_{j} \cdot \mathbf{E} d S
$$

where $\boldsymbol{\tau}_{j}$ is the unit tangent to the edge $e_{j}$, as shown in Figure 1.

To avoid confusion with the exact electric field $\mathbf{E}$ we define the vector of degrees of freedom as

$$
\mathbf{U}:=\left[E_{j}: 1 \leq j \leq N\right]^{T},
$$

where $N$ is the global number of degrees of freedom. Since the magnetic field $H$ is defined

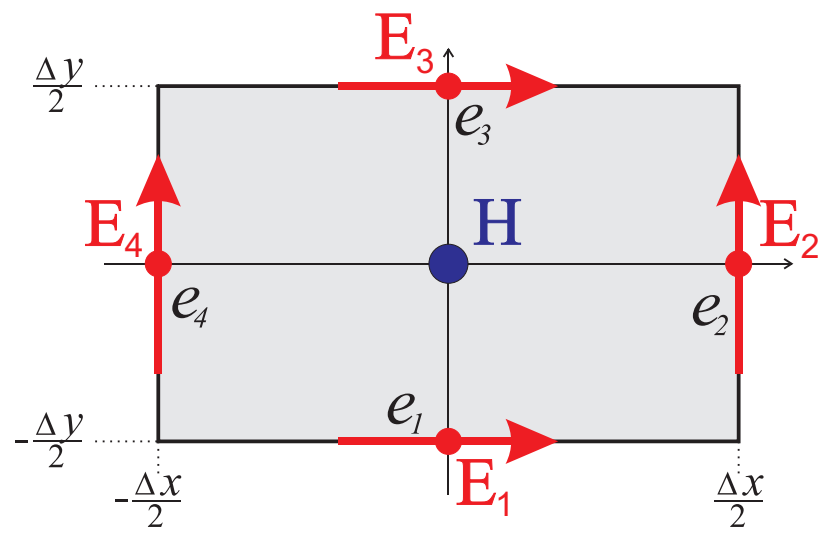

Figure 1: Illustration of the degrees of freedom on a rectangular element.

only by an average value on each element, we will assume that $H$ is constant on each 
element. Just like for the continuous equations, in the discrete case the magnetic field $H$ can be eliminated from the equations. We mention the discrete representation of $H$ because our construction of the stiffness matrix will rely on it in Section 3.4.

The electric field is defined by at least three d.o.f. on each element. The number of d.o.f. depends on the number of edges in the polygonal element. On a triangular element the discrete approximation space $V_{h}$ is the classical three-dimensional approximation space:

$$
\mathbf{v}_{1}=\left[\begin{array}{l}
1 \\
0
\end{array}\right], \quad \mathbf{v}_{2}=\left[\begin{array}{l}
0 \\
1
\end{array}\right], \quad \mathbf{v}_{3}=\left[\begin{array}{r}
-y \\
x
\end{array}\right]
$$

Remark. The local approximation space, being a linear space, admits many different bases. The standard choice for FE discretizations is the Lagrangian basis which, indeed, satisfies $d_{i}\left(\mathbf{v}_{j}\right)=\delta_{i j}$. This choice of basis is associated with the d.o.f. and is convenient for the assembly of global matrices. For our construction it is convenient to use a different local basis containing monomials, see equation (3.3). After computing the mass and the stiffness matrices $\widetilde{M}^{P}$ and $\widetilde{A}^{P}$ in this basis we then convert them to the standard Lagrangian basis using the transformation (3.5a-3.5b). We will also deviate from the standard d.o.f. for Nédélec Elements and use average values of tangential components over each edge instead of tangential integrals to improve the scaling of our d.o.f. matrix and to conform to the standard convention for MFD, c.f. [17].

On a rectangular element the approximation space (3.3) is enriched by an additional (fourth) basis function. The choice of this basis function will vary with the method and will be discussed in each of the Sections 3.2, 3.3, and 3.4.

Before diverging in our presentation of the three different methods, we present the common framework. Let $\left\{\mathbf{v}_{j}\right\}_{j=1}^{n} \subset \mathbf{H}($ curl $)$ be the basis of the discrete approximation space $V_{h}$ on the element. Let $\left[T_{i j}\right]$ be a matrix of d.o.f. for the basis functions $\mathbf{v}_{i}$. Then the matrices $T=\left[T_{i j}\right]$ and $T^{-1}$ represent the change of representation from the basis $\mathbf{v}_{i}$ to the basis of d.o.f. First, we define the local mass and stiffness matrices $\widetilde{M}^{P}$ and $\widetilde{A}^{P}$ on a polygonal element $P$ in the basis $\left\{\mathbf{v}_{j}\right\}_{j=1}^{n}$

$$
\begin{aligned}
\widetilde{M}^{P} & =\left[\widetilde{m}_{i j}\right], & \widetilde{m}_{i j} & =\int_{P} \mathbf{v}_{i} \cdot \mathbf{v}_{j} d A \\
\widetilde{A}^{P} & =\left[\widetilde{a}_{i j}\right], & \widetilde{a}_{i j} & =\int_{P}\left(\operatorname{curl} \mathbf{v}_{i}\right)\left(\operatorname{curl}_{j}\right) d A
\end{aligned}
$$

Next, to define the local matrices in the basis associated with d.o.f. we perform a "change of basis"

$$
\begin{aligned}
M^{P} & =T^{-T} \widetilde{M}^{P} T^{-1}, \\
A^{P} & =T^{-T} \widetilde{A}^{P} T^{-1} .
\end{aligned}
$$

Assembly of the local matrices $M^{P}$ and $A^{P}$ into a global matrices $M$ and $A$ respectively is done in the usual way. Using a central finite difference discretization of the time-derivative term with time step $\Delta t$ we obtain a fully discrete representation of (2.2) given appropriate 
initial conditions $\mathbf{U}^{0}$ and $\mathbf{U}^{-1}$, we have

$$
M\left(\mathbf{U}^{j+1}-2 \mathbf{U}^{j}+\mathbf{U}^{j-1}\right)=-c^{2} \Delta t^{2} A \mathbf{U}^{j}
$$

where the superscript denotes the time level.

\subsection{Efficient treatment of the mass matrix}

Time integration of the numerical scheme (3.6) requires a linear solve for $U^{j+1}$ at every time-step. There are two common ways of avoiding (or drastically simplifying) the linear solve.

One commonly used approach is called mass-lumping. In this approach the mass matrix is approximated by a diagonal matrix $D \approx M$, obtained by moving ("lumping") all entries of $M$ to the diagonal:

$$
D_{i j}=\delta_{i j} \sum_{k=1}^{n} M_{i k} .
$$

This does not change the accuracy order of the scheme, but in case of the acoustic and Maxwellian waves decreases the accuracy by increasing the constant in front of the error at the highest order [21].

Another, more recent, approach is to take the mass-lumping idea a step further:

$$
M \approx D=D I \approx D M^{-1} D:=W^{-1} \quad \Rightarrow \quad M^{-1} \approx D^{-1} M D^{-1}=W .
$$

Typically, this approach by itself does not yield significant benefits over simple mass-lumping (3.7). The advantages of (3.8) over (3.7) can be realized if one can further modify the mass matrix $M$ on the right of (3.8). This will be the idea in Sections 3.3 and 3.4, that will ultimately lead to a dramatic reduction of numerical dispersion.

For all of the forthcoming methods it can be shown that the local lumped matrix $D^{P}$

$$
D_{i j}^{P}=\delta_{i j} \sum_{j=1}^{4} M_{i j}=\frac{|P|}{2} I_{4 \times 4}
$$

where $I_{m \times m}$ is the $m \times m$ identity matrix and $|P|$ is the area of of the polygon $P$. If $\mathcal{Q}$ is the quadrangulation of our domain and $A_{P \in \mathcal{Q}}$ is the assembly operator then

$$
D=\mathrm{A}_{P \in \mathcal{Q}} D^{P}=|P| I_{N \times N},
$$

as each degree of freedom in $D$ occurs in exactly two elements. From this identity we can express the global matrix $W$ as a scalar multiple of the matrix $M$ as follows:

$$
W=|P|^{-2} M
$$

This suggests that if $W=\mathrm{A}_{P \in \mathcal{Q}} W^{P}$ then

$$
W^{P}=|P|^{-2} M^{P}
$$


For all forthcoming methods we use the following fully explicit, full discrete scheme:

$$
\mathbf{U}^{j+1}=2 \mathbf{U}^{j}-\mathbf{U}^{j-1}-c^{2} \Delta t^{2} W A \mathbf{U}^{j} .
$$

Using the Equation (3.10) we can rewrite the above scheme in terms of the Courant number $\nu$ :

$$
\mathbf{U}^{j+1}=2 \mathbf{U}^{j}-\mathbf{U}^{j-1}-\nu^{2}\left(|P|^{-1} M\right) A, \quad \nu^{2}=\frac{c^{2} \Delta t^{2}}{|P|} .
$$

This is useful as the matrix $\left(|P|^{-1} M\right) A$ scales like a constant and the Courant number, which is frequently crucial to stability, appears explicitly.

\subsection{Nédélec's edge elements}

In this section we present Nédélec's edge based elements. Consider a rectangular element $[-\Delta x / 2, \Delta x / 2] \times[-\Delta y / 2, \Delta y / 2]$, as shown on Figure 1. The approximation space is given by the basis functions:

$$
\mathbf{v}_{1}=\left[\begin{array}{l}
1 \\
0
\end{array}\right], \quad \mathbf{v}_{2}=\left[\begin{array}{l}
0 \\
1
\end{array}\right], \quad \mathbf{v}_{3}=2\left[\begin{array}{c}
-\frac{y}{\Delta y} \\
\frac{x}{\Delta x}
\end{array}\right], \quad \mathbf{v}_{4}=2\left[\begin{array}{c}
\frac{y}{\Delta y} \\
\frac{x}{\Delta x}
\end{array}\right] .
$$

Here the scaling for the functions $\mathbf{v}_{3}$ and $\mathbf{v}_{4}$ is selected for convenience, to make the columns $T_{j}$ of the transformation matrix $T$, defined by the d.o.f. of $\mathbf{v}_{j}$, independent of the dimensions of the element:

$$
T=\left[\begin{array}{rrrr}
1 & 0 & 1 & 1 \\
0 & 1 & 1 & -1 \\
1 & 0 & -1 & -1 \\
0 & 1 & -1 & 1
\end{array}\right] .
$$

Based on the definition of the basis functions (3.14), in the case of $\Delta x=\Delta y=h$, one can compute local mass and stiffness matrices in the basis $\mathbf{v}_{i}$, exactly:

$$
\begin{gathered}
\widetilde{M}^{P}=h^{2}\left[\begin{array}{cccc}
1 & 0 & 0 & 0 \\
0 & 1 & 0 & 0 \\
0 & 0 & 2 / 3 & 0 \\
0 & 0 & 0 & 2 / 3
\end{array}\right], M^{P}=\frac{h^{2}}{6}\left[\begin{array}{llll}
2 & 0 & 1 & 0 \\
0 & 2 & 0 & 1 \\
1 & 0 & 2 & 0 \\
0 & 1 & 0 & 2
\end{array}\right], W^{P}=\frac{1}{6 h^{2}}\left[\begin{array}{llll}
2 & 0 & 1 & 0 \\
0 & 2 & 0 & 1 \\
1 & 0 & 2 & 0 \\
0 & 1 & 0 & 2
\end{array}\right], \\
\widetilde{A}^{P}=\left[\begin{array}{cccc}
0 & 0 & 0 & 0 \\
0 & 0 & 0 & 0 \\
0 & 0 & 16 & 0 \\
0 & 0 & 0 & 0
\end{array}\right], \text { and } A^{P}=\left[\begin{array}{rrrr}
1 & 1 & -1 & -1 \\
1 & 1 & -1 & -1 \\
-1 & -1 & 1 & 1 \\
-1 & -1 & 1 & 1
\end{array}\right]
\end{gathered}
$$

Note, that the auxiliary matrices $\widetilde{M}^{P}$ and $\widetilde{A}^{P}$ are written in a special basis $\left\{\mathbf{v}_{j}\right\}_{j=1}^{n}$ The mass, $M^{P}$, and the stiffness, $A^{P}$, matrices written in the (standard) d.o.f. basis are expressed in terms of $\widetilde{M}^{P}$ and $\widetilde{A}^{P}$ using change of basis (3.5a-3.5b) with the transformation matrix (3.15). This last operation changes diagonal matrices $\widetilde{M}^{P}$ and $\widetilde{A}^{P}$ to the non-diagonal ones, $M^{P}$ and $A^{P}$. Further the matrix $M^{P}$ is auxiliary to the local approximate mass inverse $W^{P}$ which is computed using the equation $W^{P}=h^{-4} M^{P}$. The global discretization is obtained by assembling local matrices $W^{P}$ and $A^{P}$. 


\subsection{Guddati-Yue's family of 4-point quadratures}

In this section we use the ideas of Guddati and Yue first proposed for acoustics wave propagation, [8]. In this method the approximation space is the same as in the Nédélec's edge elements, see (3.14). However, instead of computing local mass and stiffness matrices exactly, as it was done in (3.16), they are computed approximately using a 4-point quadrature.

These quadratures are defined by a two parameters, $a_{M}$ and $a_{A}$, indicating the position of the quadrature points for the mass and the stiffness matrices, respectively:

$$
p_{j}=\left( \pm a_{*} \frac{\Delta x}{2}, \pm a_{*} \frac{\Delta y}{2}\right) \quad *=M \text { or } A .
$$

The quadrature weights are chosen to be the same, producing the following quadratures

$$
Q(f)=\frac{h^{2}}{4} \sum_{j=1}^{4} f\left(p_{j}\right)
$$

On rectangular meshes, all such quadratures $\left(a_{*} \neq 0\right)$ approximate integrals of linear functions exactly.

Remark. As it turns out, unlike in the case of acoustics, in the case of Maxwell's equations the stiffness matrix does not depend on the choice of the stiffness parameter $a_{A}$. This is because all basis functions $\mathbf{v}_{i}, i=1, \ldots, 4$, in case of the Maxwell's equations are linear and their derivatives constants. Hence, the use of quadrature does not introduce any ambiguity for computing the energy integrals. In case of the acoustics, the fourth basis functions was bilinear. Hence, its derivative is linear and the integral of linear functions with itself using the above four-point quadrature rule is not exact. Therefore, in the case of the acoustics equations, there was a difference as to which of the quadrature points $a_{A}$ should be used.

The mass matrix, unlike the stiffness matrix, depends on the parameter $a_{M}$. Here is the dependence for the local mass matrix written in the basis $\mathbf{v}_{i}$ :

$$
\widetilde{M}^{P}=h^{2}\left[\begin{array}{cccc}
1 & 0 & 0 & 0 \\
0 & 1 & 0 & 0 \\
0 & 0 & 2 a_{M}^{2} & 0 \\
0 & 0 & 0 & 2 a_{M}^{2}
\end{array}\right] .
$$

This construction produces a one parameter family of discretizations parametrized by the mass matrix quadrature parameter $a_{M}$. As before, the local matrices in terms of d.o.f. are obtained using the change of basis transformation (3.5a-3.5b) with the transformation matrix (3.15). Again, the global discretization is obtained by assembling local matrices $W^{P}$ and $A^{P}$ into global ones, $W$ and $A$.

\subsection{Mimetic finite differences}

In this section we consider a Mimetic Finite Difference (MFD) discretization, which can be seen as a generalization of the Guddati and Yue approach in the sense that the MFD family is richer and contains the GY family. Since the family of schemes is richer, it is not 
surprising that through an optimization of parameters we can obtain a scheme with better dispersion characteristics. We will now introduce the family.

Consider the approximation space given by the basis functions

$$
\mathbf{v}_{1}=\left[\begin{array}{l}
1 \\
0
\end{array}\right], \quad \mathbf{v}_{2}=\left[\begin{array}{l}
0 \\
1
\end{array}\right], \quad \mathbf{v}_{3}=\left[\begin{array}{r}
-\frac{y}{\Delta y} \\
\frac{x}{\Delta x}
\end{array}\right], \quad \mathbf{v}_{4}=\left[\begin{array}{l}
? \\
?
\end{array}\right] .
$$

The fourth basis function $\mathbf{v}_{4}$ was intentionally left undefined, as its exact definition will not be important in this context.

Let us define the scalar curl operator that operates from the space of vector electric field $\mathbf{E}$, defined by four values per element, to the space of magnetic field $H$, defined by a single value on each element. The natural definition of curl operator is based on Stokes's Theorem

$$
\frac{1}{|P|} \int_{P} \operatorname{curl}(\vec{u}) d A=\frac{1}{|P|} \int_{\partial P} \vec{u} \cdot \vec{\tau} d s=\frac{1}{|P|} \sum_{e_{i}} \vec{u} \cdot \vec{\tau}_{i}=\frac{1}{|P|} \sum_{e_{i}} o\left(e_{i}\right)\left|e_{i}\right| u_{i} .
$$

Here $P$ is the area of the polygonal element, $\left|e_{i}\right|$ is the length of the edge $e_{i}, o\left(e_{i}\right)= \pm 1$ is the orientation of the edge, and $u_{i}$ is the d.o.f. - the average value of $\vec{u}$ along the edge $e_{i}$. Assuming enumeration of edges as shown on Figure 1, the orientation function is defined to be

$$
o\left(e_{1}\right)=1, \quad o\left(e_{2}\right)=1, \quad o\left(e_{3}\right)=-1, \quad o\left(e_{4}\right)=-1 .
$$

Based on (3.22), the discrete $\mathrm{curl}_{h}$ operator is defined locally as

$$
\operatorname{curl}_{h}\left[\begin{array}{c}
u_{1} \\
u_{2} \\
u_{3} \\
u_{4}
\end{array}\right]=\left[\begin{array}{c}
o\left(e_{1}\right)\left|e_{1}\right| \\
o\left(e_{2}\right)\left|e_{2}\right| \\
o\left(e_{3}\right)\left|e_{3}\right| \\
o\left(e_{4}\right)\left|e_{4}\right|
\end{array}\right]^{T}\left[\begin{array}{l}
u_{1} \\
u_{2} \\
u_{3} \\
u_{4}
\end{array}\right]=\left[\begin{array}{r}
\Delta x \\
\Delta y \\
-\Delta x \\
-\Delta y
\end{array}\right]^{T}\left[\begin{array}{l}
u_{1} \\
u_{2} \\
u_{3} \\
u_{4}
\end{array}\right] .
$$

The local (discrete) $\mathrm{curl}_{h}$ operator acts from cell edges to cell centers. Therefore, locally, it can be represented by a $1 \times 4$ matrix. The global representation of curl $_{h}$ can be obtained by the standard assembly process. The stiffness matrix is then defined in terms of the discrete $\operatorname{curl}_{h}$ operator and the product matrix for the magnetic field $H_{h}$. The latter is just a scalar $|P|$ on each element. Therefore, the stiffness matrix takes the form

$$
A^{P}=\operatorname{curl}_{h}^{T}|P| \operatorname{curl}_{h}
$$

It is a technical exercise to check that the representation (3.24), written in the d.o.f., matches with the construction of the stiffness matrix in (3.16) after the change of basis procedure (3.5a-3.5b) with the transformation matrix (3.15). Thus, just like in Section 3.3 the stiffness is defined uniquely.

The local mass matrix is constructed, in such a way that it approximates the integrals of constants exactly, and allows for an error in the approximation of the products of higher order functions. 
In the local basis $\mathbf{v}_{i}$ the mass matrix has the form

$$
\widetilde{M}^{P}=\Delta x \Delta y\left[\begin{array}{cc|cc}
1 & 0 & 0 & 0 \\
0 & 1 & 0 & 0 \\
\hline 0 & 0 & m_{1} & m_{2} \\
0 & 0 & m_{2} & m_{3}
\end{array}\right] .
$$

The top left block of the matrix represents the products of constants and it is computed exactly. The off-diagonal blocks are selected to be zero so as to indicate the $L^{2}$ orthogonality of the basis functions. This is a typical MFD construction, but it may not be necessary for preserving the order of the scheme. The bottom right block, is the block representing products of the non-constant basis functions, which is represented approximately.

The values of the parameters $m_{1}, m_{2}, m_{3}$ can be varied as long as two conditions are satisfied:

- The bottom right sub-matrix of (3.25) is symmetric (always true due to notations) and positive definite (may not be true based on the choice of $m_{i}$ ).

- The bottom right sub-matrix of (3.25) scales like the top left sub-matrix of (3.25).

The above conditions are necessary for the stability of the scheme.

Remark. The construction of the mass matrix (3.25), in a way, is a generalization of the quadrature idea, where higher-order error is introduced which does not change the formal accuracy of the scheme. The bottom right block of the mass matrix (3.25) contains the analogue of the quadrature error. The small error then can be optimized to improve the dispersion properties of the scheme.

We have the following inclusion of the families of schemes

$$
\text { Nédélec } \in \text { Guddati-Yue } \subset \text { MFD. }
$$

The larger is the family of the schemes, the higher is its flexibility to improve the dispersion properties.

\section{Dispersion Relationship}

Fourier modes form a basis in $L^{2}$ and many of its subspaces. Moreover, linear combinations of planar Fourier waves

$$
f(x, t)=\operatorname{expi}(\mathbf{k} \cdot \mathbf{x}-\omega t)
$$

are dense in these spaces. Arising in classical Fourier analysis, a dispersion relationship is the functional relationship between spatial wave numbers $\mathbf{k}$ and temporal frequencies $\omega=\omega(\mathbf{k})$ for those plane waves which solve a particular linear, Cauchy PDE. For linear PDEs the dispersion analysis allows us to understand the qualitative behavior of a solution as a superposition of planar wave modes (4.1). The dispersion analysis can be applied to the continuous PDEs and their discretizations alike.

We will start with the dispersion analysis of the continuous PDE (2.2) and then perform the dispersion analysis for the numerical schemes. 


\subsection{The dispersion relation for the continuous PDE}

As typically done, we ignore the boundary conditions by considering the solution in the whole of $\mathbb{R}^{2} \times \mathbb{R}^{+}$. We search for solutions of (2.2) in the planar wave form

$$
\mathbf{E}(x, t)=E_{0} \mathbf{u} \exp (\mathrm{i} k \mathbf{k} \cdot \mathbf{x}-\mathrm{i} \omega t), \quad|\mathbf{u}|=|\mathbf{k}|=1,
$$

which we substitute into the PDE (2.2)

$$
E_{0} \frac{\partial^{2}}{\partial t^{2}} \mathbf{u} e^{\mathrm{i}(k \mathbf{k} \cdot \mathbf{x}-\omega t)}=-c^{2} E_{0} \operatorname{curlcurl} \mathbf{u} e^{\mathrm{i}(k \mathbf{k} \cdot \mathbf{x}-\omega t)} .
$$

We then simplify the above expression using properties of the exponential

$$
E_{0}(-\mathrm{i} \omega)^{2} \mathbf{u} e^{\mathrm{i}(k \mathbf{k} \cdot \mathbf{x}-\omega t)}=-c^{2} E_{0}(\mathrm{i} k)^{2} \mathbf{k} \times \mathbf{k} \times \mathbf{u} e^{\mathrm{i}(k \mathbf{k} \cdot \mathbf{x}-\omega t)} .
$$

Canceling the exponential terms and initial amplitudes, leaves us with the dispersion relationship between the wave number, $k \mathbf{k}$, and the frequency, $\omega$ :

$$
\omega^{2} \mathbf{u}=c^{2} k^{2}((\mathbf{k} \times \mathbf{u}) \times \mathbf{k}) .
$$

We include the following classical result without proof.

Lemma 4.1. The dispersion relation (4.5) allows for a solution $\omega$ if and only if $\mathbf{k} \perp \mathbf{u}$ or $\mathbf{k} \| \mathbf{u}$. The corresponding solutions are

$$
\omega^{2}=c^{2} k^{2} \quad \text { for } \quad \mathbf{k} \perp \mathbf{u} \quad \text { and } \quad \omega^{2}=0 \text { for } \quad \mathbf{k} \| \mathbf{u} .
$$

Remark. For a planar wave (4.2), the conservation condition $\nabla \cdot \mathbf{E}=0$ is equivalent to the orthogonality condition $\mathbf{k} \perp \mathbf{u}$, which is the first condition in (4.6). The second condition in (4.6) correspond to a standing wave.

\subsection{The dispersion relation for numerical schemes}

Let $\Delta x$ and $\Delta y$ be the spacial mesh resolutions and $\Delta t$ the space discretization step. Let $M, M^{P}$ and $A, A^{P}$ be global and local mass and stiffness matrices, respectively (written in the d.o.f. basis). Just like in the case of the continuous PDE, we seek a planar solution as in equation (4.2).

To obtain a dispersion relation, we need to construct a discrete representation of the function (4.2) and substitute it into the numerical scheme (3.12). We do so in the following section where we develop some auxiliary results to simply the dispersion analysis.

\subsubsection{Auxiliary construction for the discrete waveforms}

Let $P$ be one of the elements in the mesh. Let $u_{1}$ and $u_{2}$ be the d.o.f. of the wave (4.2) corresponding to the edges $e_{1}$ and $e_{2}$, as shown on Figure 1. Then all degrees of freedom for the wave (4.2) can be written in terms of $u_{1}, u_{2}$ and the exponent $e^{\mathbf{i}\left(\mathbf{k} \cdot \Delta \mathbf{x}_{\mathbf{i}}\right)}$ as

$$
\begin{array}{ll}
u_{i}=u_{1} e^{\mathrm{i}\left(\mathbf{k} \cdot \mathbf{\Delta} \mathbf{x}_{\mathbf{i}}\right)} & \text { for a horizontal edge } e_{i}, \\
u_{i}=u_{2} e^{\mathrm{i}\left(\mathbf{k} \cdot \mathbf{\Delta} \mathbf{x}_{\mathbf{i}}\right)} & \text { for a vertical edge } e_{i},
\end{array}
$$


where $\Delta \mathbf{x}_{\mathbf{i}}$ is the "shift"-vector from the center of the edge $u_{1}$ or $u_{2}$ to the center of the edge $e_{i}$, respectively. In particular, the four d.o.f. $\left(u_{1}, u_{2}, u_{3}, u_{4}\right)$, corresponding to the element $P$, can be written in terms of the first two $\left(u_{1}, u_{2}\right)$ as

$$
\left[\begin{array}{l}
u_{1} \\
u_{2} \\
u_{3} \\
u_{4}
\end{array}\right]=S\left[\begin{array}{l}
u_{1} \\
u_{2}
\end{array}\right], \quad \text { where } \quad S:=\left[\begin{array}{cc}
1 & 0 \\
0 & 1 \\
e^{\mathrm{i} k_{2} \Delta y} & 0 \\
0 & e^{\mathrm{i} k_{1} \Delta x}
\end{array}\right] .
$$

From (4.7) one can see that the global dispersion relation can be expressed only in terms of two d.o.f., $u_{1}$ and $u_{2}$. In order to produce a simple form of the dispersion relation we first consider multiplication of a waveform (4.7) by a global matrix $X$ assembled from generic local matrices $X^{P}$ that are the same for all elements. In our numerical scheme (3.12) we perform such multiplications twice; first, by a stiffness matrix $A$, followed by the mass-related matrix $W$. To proceed we require the following result.

Lemma 4.2. Consider the result of multiplication

$$
V=X U,
$$

where the matrix $X$ is assembled from identical local matrices $X^{P}$ and the vector of d.o.f. $U$ is given by (4.7). Then the vector of d.o.f. $V$ also satisfies the relation (4.7), where all u's are replaced by $v$ 's. The two d.o.f. $\left(v_{1}, v_{2}\right)$ defining the vector $V$ depend linearly on the two degrees of freedom $\left(u_{1}, u_{2}\right)$ defining the vector $U$; the corresponding two-by-two matrix has the form $\left(S^{*} X^{P} S\right)$ :

$$
\left[\begin{array}{l}
v_{1} \\
v_{2}
\end{array}\right]=\left(S^{*} X^{P} S\right)\left[\begin{array}{l}
u_{1} \\
u_{2}
\end{array}\right],
$$

where $S^{*}$ is a conjugate transpose of $S$.

Proof. The fact that $V$ satisfies (4.7) follows immediately from the fact that $U$ satisfies (4.7). The linear relation between $\left(v_{1}, v_{2}\right)$ and $\left(u_{1}, u_{2}\right)$ is a direct consequence of the linear relation (4.9). The main point of the lemma is to show that the linear relation (4.10) is given by the two-by-two matrix $\left(S^{*} X^{P} S\right)$.

Consider the two elements $P_{1}$ and $P_{2}$ that determine the value of $v_{1}$ and the two elements $P_{1}$ and $P_{3}$ that determine the values of $v_{2}$, as shown on Fig. 2. The value of $v_{1}$ is a sum of the contributions from the elements $P_{1}$ and $P_{2}$

$$
\begin{aligned}
v_{1} & =\left[\begin{array}{llll}
1 & 0 & 0 & 0
\end{array}\right] X^{P} S\left[\begin{array}{l}
u_{1} \\
u_{2}
\end{array}\right]+\left[\begin{array}{llll}
0 & 0 & 1 & 0
\end{array}\right] X^{P} S\left[\begin{array}{l}
e^{-\mathrm{i} k_{2} \Delta y} u_{1} \\
e^{-\mathrm{i} k_{2} \Delta y} u_{2}
\end{array}\right]= \\
& =\left[\begin{array}{llll}
1 & 0 & e^{-\mathrm{i} k_{2} \Delta y} & 0
\end{array}\right] X^{P} S\left[\begin{array}{l}
u_{1} \\
u_{2}
\end{array}\right] .
\end{aligned}
$$

Here the product $X^{P} S\left(u_{1}, u_{2}\right)^{T}$ is a size four vector of contributions from the element to the four d.o.f. of $v$, while multiplication on the left by $(1,0,0,0)$ extracts the first component of this vector. 


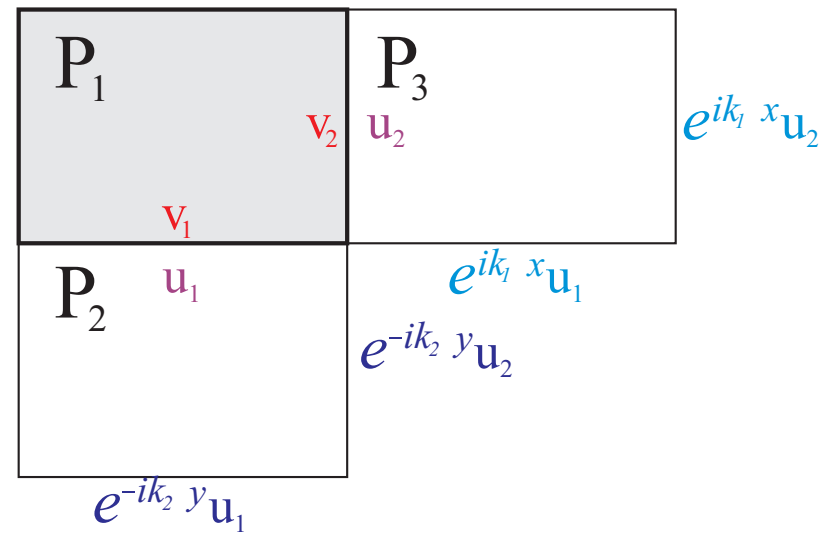

Figure 2: Three elements that are involved in the definition of values of $v_{1}$ and $v_{2}$.

Similarly, to (4.11) we get the expression for $v_{2}$

$$
\begin{aligned}
v_{2} & =\left[\begin{array}{llll}
0 & 1 & 0 & 0
\end{array}\right] X^{P} S\left[\begin{array}{l}
u_{1} \\
u_{2}
\end{array}\right]+\left[\begin{array}{llll}
0 & 0 & 0 & 1
\end{array}\right] X^{P} S\left[\begin{array}{c}
e^{-\mathrm{i} k_{1} \Delta x} u_{1} \\
e^{-\mathrm{i} k_{1} \Delta x} u_{2}
\end{array}\right]= \\
& =\left[\begin{array}{llll}
0 & 1 & 0 & e^{-\mathrm{i} k_{1} \Delta x}
\end{array}\right] X^{P} S\left[\begin{array}{l}
u_{1} \\
u_{2}
\end{array}\right] .
\end{aligned}
$$

Combining (4.11) and (4.12) and recalling the definition (4.8) of the transformation matrix $S$ we obtain the final result (4.10).

\subsubsection{The discrete dispersion relation}

Using the tools from the Section 4.2.1 we are now ready to formulate the discrete dispersion relationship in a compact form. This and similar types of analysis can be found in $[1,3,5,10]$ and in references therein. First, substitute the plane wave ansatz (4.2) into the discrete scheme (3.13) and cancel the time-dependent exponential term present on both sides:

$$
2\left(\cos \left(\omega_{n} \Delta t\right)-1\right) \mathbf{U}=-\nu^{2}\left(|P|^{-1} M\right) A \mathbf{U}, \quad \nu=\frac{c \Delta t}{h} .
$$

We then pose the discrete dispersion relationship as a global eigenvalue problem

$$
\lambda \mathbf{U}=|P|^{-1} M A \mathbf{U} \quad \text { where } \quad \lambda=\frac{2\left(1-\cos \left(\omega_{n} \Delta t\right)\right)}{\nu^{2}} .
$$

Here $\omega_{n}$ is the numerical frequency. We find it useful to consider $\omega_{n}=c_{n} k$ and rewrite the cosine term of the above equation, recalling that $\nu h=c \Delta t$ as follows:

$$
\cos \left(\omega_{n} \Delta t\right)=\cos \left(\frac{c_{n}}{c} \nu k h\right)
$$

Now, we use the result of Lemma 4.2 twice, first for $A$ then for $M$ to rewrite the relation (4.14) only in terms of two d.o.f. $\mathbf{U}^{P}=\left(u_{1}, u_{2}\right)^{T}$ :

$$
\lambda \mathbf{U}^{P}=\bar{M}^{P} \bar{A}^{P} \mathbf{U}^{P}, \quad \text { where } \quad \bar{M}^{P}=|P|^{-1} S^{*} M^{P} S \quad \text { and } \quad \bar{A}^{P}=S^{*} A^{P} S .
$$


The equation (4.16) is a discrete dispersion relation posed as an eigenvalue problem, just as in the classical case.

\subsection{Phase error}

We will now transition from the case of rectangular discretizations to the case of a square mesh. In case the spatial parameter $|P|=h^{2}$ where $h$ is the side length of a square. The numerical speed of the wave with frequency $\omega_{n}$ is $c_{n}=\frac{\omega_{n}}{k}$, similarly to the physical numerical speed $c=\frac{\omega}{k}$. The difference between the numerical and physical wave speeds is that the physical speed is constant independent of the wave-number $k$ and other variables, while the numerical speed $c_{n}$ varies depending on the wave-length $2 \pi / k$ relative to the mesh size $h$ and the direction of propagation of the wave. The ratio of the wave-length $2 \pi / k$ to the mesh size $h$ is the number of grid points per wavelength $\left(N_{\text {p.p.w. }}\right)$

$$
\frac{2 \pi}{k h}=N_{p . p . w .}
$$

We take $k h$ to be our primary wave resolution parameter.

We will be interested in accurately recovering $c_{n}$ in the limit of small $k h$ (many points per wavelength). For this purpose, consider a Taylor series expansion of $c_{n}$ :

$$
c_{n}=c\left(C_{0}+C_{1}(k h)+C_{2}(k h)^{2}+C_{3}(k h)^{3}+\ldots\right) .
$$

The numerical scheme is consistent if $C_{0}=1$. This will be the case for all of the methods considered here. We define the relative phase error of the scheme as the quantity

$$
\varphi_{n}=\frac{\left|c_{n}-c\right|}{|c|}=C_{1}(k h)+C_{2}(k h)^{2}+\ldots
$$

We will say that the numerical scheme has $n$-th order numerical phase error if $C_{0}=1$ and $C_{n}$ is the first nonzero coefficient in (4.18). We will say that the numerical scheme has $n$-th order numerical anisotropy if $C_{n}$ is the first coefficient in (4.18) that depends on the direction of the wave. Clearly, the numerical anisotropy order is at least the numerical dispersion order.

\section{Dispersion Analysis and Adaptation}

In this section we will compute the numerical phase error of the three classes of discretization. We will select a member the GY family and MFD family which reduce the numerical phase error for each of the methods. We call this process GY- or $M$-adaptation respectively. For the following three sections we will take $\mathbf{k}=[\cos \theta, \sin \theta]^{T}$, where $\theta$ is the angle between the direction of propagation and the $x$-axis of the mesh.

\subsection{Nédélec's discretization}

In this section we will show that the Nédélec discretization has second order phase error and numerical anisotropy. 
Theorem 5.1. The Nédélec discretization has second order numerical phase error and second order numerical anisotropy.

Proof. Consider the dispersion relation (4.16) and take a Taylor expansion of both sides in the small parameter $k h$. To perform the expansion, we first need to use the definition (4.14) of the eigenvalue $\lambda$. Next we rewrite the component of $\lambda$ in a form that explicitly contains the small parameter $k h$ as in (4.15):

$$
\cos \left(\omega_{n} \Delta t\right)=\cos \left(c_{n} k \Delta t\right)=\cos \left(\frac{c_{n}}{c} k h \frac{c \Delta t}{h}\right)=\cos \left(\frac{c_{n}}{c} k h \nu\right),
$$

where the numerical wave speed $c_{n}$ has the form (4.18). Matching the terms at the same order of $k h$ we find:

$$
C_{0}=1, \quad C_{1}=0, \quad C_{2}=\frac{1}{96}\left(\left(4 \nu^{2}-9\right)-3 \cos 4 \theta\right) .
$$

Since $C_{2}$ is the first non-zero term in (4.18) and since it further has angular dependence the numerical dispersion and the numerical anisotropy are both of the second order.

Figure 3 (left) shows the rate of convergence of the Nédélec method for many angles. Note that the width of the band illustrates over how many orders of magnitude the numerical anisotropy varies. Figure 3 (right) shows that the lowest dispersion errors for this method occur along the diagonal.

\subsection{GY-adaptation}

Having established the Nédélec discretization as a baseline, we now identify the member of the GY family which has minimal phase error.

Theorem 5.2 (GY-adaptation). An optimal member of the GY family is obtained by taking the mass matrix parameter $a_{M}=\frac{2}{\sqrt{3}}$. The corresponding method has second order numerical phase error and fourth order numerical anisotropy.

Proof. Performing the same steps as in Section 5.1 now for the GY family of scheme, derived in Section 3.3, we obtain an expression for the largest eigenvalue in (4.16). This leads to the following dependence of the error terms in (4.18) on the quadrature parameter $a_{M}$ :

$$
C_{0}=1, \quad C_{1}=0, \quad C_{2}=\frac{1}{96}\left(\left(4 \nu^{2}+9 a_{M}^{2}-12\right)+\left(3 a_{M}^{2}-4\right) \cos (4 \theta)\right) .
$$

No choice of a constant value of the parameter $a_{M}$ makes $C_{2}=0$. The best we can hope to do is eliminate the dependence of the second order term $C_{2}$ on the direction of the wave $\theta$. This can be done by taking $a_{M}=\frac{2}{\sqrt{3}}$. Using this value of the quadrature parameter the following terms in the expansion (4.18) become

$$
C_{2}=\frac{\nu^{2}}{24}, \quad C_{3}=0, \quad C_{4}=\frac{1}{5760}\left(\left(27 \nu^{2}-20\right)-12 \cos 4 \theta\right)
$$

Therefore, the optimal member of the GY family has second order numerical dispersion and fourth order numerical anisotropy. 
Remark. This analysis shows that for the optimal method described above, dispersion error can be reduced by taking $\nu$ small. This is in contrast to methods like the Yee-Scheme where the lowest relative phase error is attained when $\nu$ is as large as possible.

In Figure 3 (left) we see that the optimal GY member has second-order phase error just like the Nédélec discretization. However, the anisotropic band of this method is completely contained inside the Nédélec band. Further, the anisotropy is perhaps best illustrated by Figure 3 (right) where the relative dispersion error for the method appears completely circular while still being on the same order of magnitude as the Nédélec method.

Remark. Often the anisotropy of a numerical scheme is visualized using the ratio $\frac{c_{n}}{c}$, see e.g., [6]. This is convenient for showing the differences between schemes but it does not capture well the order of magnitude differences in anisotropy between them. To highlight the latter we plot a related quantity, $\left|1-\frac{c_{n}}{c}\right|$, on a logarithmic scale, see Figure 3 (right).

\subsection{M-adaptation}

In this section, similarly to the Section 5.2, we first find the optimal member of the MFD family. We then find the stability region for the optimal member.

Theorem 5.3 (M-adaptation). The optimal member of the MFD family has fourth order numerical phase error and fourth order numerical anisotropy.

Proof. For the MFD family derived in Section 3.4 the leading order error terms in the expansion (4.18) are

$$
\begin{aligned}
& C_{0}=1, \quad C_{1}=0, \\
& \left.C_{2}=\frac{1}{192}\left(\left(-24+8 \nu^{2}+6 m_{1}+3 m_{3}\right)-12 m_{2} \cos (2 \theta)+\left(3 m_{3}-8\right) \cos (4 \theta)\right)\right),
\end{aligned}
$$

where $m_{1}, m_{2}$ and $m_{3}$ are the parameters in the mass matrix. These parameters can be selected to eliminate the $C_{2}$ term altogether by first eliminating the angle-dependent terms through a choice of $m_{3}$ and $m_{2}$ and then eliminating the remaining angle-independent term through a choice of $m_{1}$ :

$$
m_{3}=\frac{8}{3}, \quad m_{2}=0, \quad m_{1}=\frac{4}{3}\left(2-\nu^{2}\right) .
$$

For the above parameter choices the next leading terms in (4.18) are

$$
C_{3}=0, \quad C_{4}=\frac{1}{2880}\left(-10+15 \nu^{2}-4 \nu^{4}+\left(5 \nu^{2}-6\right) \cos (4 \theta)\right) .
$$

Notice, that there is a special choice of the Courant number, i.e., $\nu^{2}=\frac{6}{5}$ that eliminates the numerical anisotropy at the fourth level. Unfortunately, as it will be clear from the stability analysis, this value of the Courant number does not guaranty stability of the scheme. 

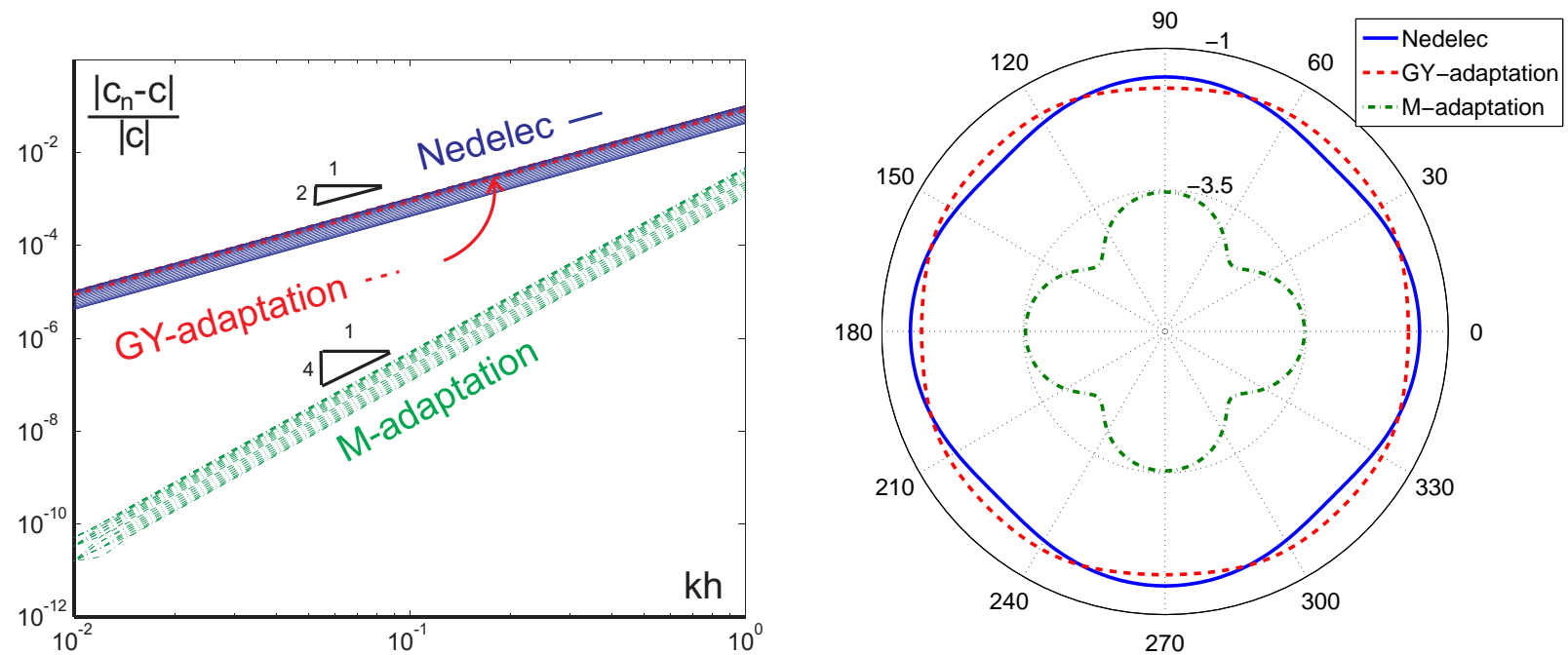

Figure 3: Shown left is a theoretical convergence plot for all three methods shown for 40 uniformly spaced angles between 0 and $\frac{\pi}{2}$ with $\log$ distribution of $k h$ between 1 and $10^{-2}$. Here anisotropy is illustrated by the width of the band. Shown right is the theoretical, relative dispersion error at twelve points per wavelength as a function of angle. Courant numbers are selected within stability regions for all three methods, namely $\nu=\frac{2}{\sqrt{3}}$ for Nédélec, $\nu=\sqrt{\frac{3}{8}}$ for GY-adapted, and $\nu=\frac{1}{\sqrt{2}}$ for M-adapted. Dispersion error is calculated from the eigenvalues of $\bar{W} \bar{A}$ and solving for $c_{n}$ using the numerical dispersion relationship.

\section{Stability Analysis}

As we have produced the discrete dispersion relationship, we can produce necessary conditions for stability using the classical technique of von Neumann.

Lemma 6.1. The derived matrices $\bar{A}^{P}, \bar{W}^{P}$ are Hermitian. One of the eigenvalues of $\bar{A}^{P}$ is zero while the other one is a non-negative real number.

Proof. Using the definitions (4.16) of the matrix $h^{-2} \bar{M}^{P}$ and recalling that $h^{-2} M^{P}$ by construction is symmetric we have

$$
\left(\bar{M}^{P}\right)^{*}=\left(S^{*} M^{P} S\right)^{*}=S^{*}\left(M^{P}\right)^{*} S=h^{-2} S^{*} M^{P} S=\bar{M}^{P} .
$$

The proof of the statement for the matrix $\bar{A}^{P}$ is identical.

Consider an eigenvalue problem (4.16). Since by construction (4.16) of the matrix $\bar{M}^{P}=$ $S^{*} M^{P} S$ it is non-singular $\left(M^{P}\right.$ is rank four and $S$ is rank two), hence, it can be inverted and its inverse is also Hermitian. Multiplying both sides of the eigenvalue equation (4.16) by $\left(\mathbf{U}^{P}\right)^{*}\left(\bar{M}^{P}\right)^{-1}$ from the left we get

$$
\lambda\left(\mathbf{U}^{P}\right)^{*}\left(\bar{M}^{P}\right)^{-1} \mathbf{U}^{P}=\left(\mathbf{U}^{P}\right)^{*} \bar{A}^{P} \mathbf{U}^{P} .
$$

Since both matrices $\left(\bar{M}^{P}\right)^{-1}$ and $\bar{A}^{P}$ are Hermitian, the two scalars $\left(\mathbf{U}^{P}\right)^{*}\left(\bar{M}^{P}\right)^{-1} \mathbf{U}^{P}$ and $\left(\mathbf{U}^{P}\right)^{*} \bar{A}^{P} \mathbf{U}^{P}$ are non-negative real numbers. Hence, the eigenvalue $\lambda$ is always non-negative.

Moreover, since $A^{P}$ is rank one the derived matrix $\bar{A}^{P}$ is at most rank one. Therefore, zero is one of the eigenvalues of (4.16). 
In the light of Lemma 6.1 we have

$$
\lambda=\operatorname{tr}\left(\bar{M}^{P} \bar{A}^{P}\right) .
$$

\subsection{Norm-based stability bounds}

In this section we derive the stability condition for a numerical scheme based on the dispersion relation (4.16).

A numerical scheme is unstable if the wave frequency $w_{n}$ has an imaginary component for some wave number $k$ and some direction of the wave. This corresponds to the term $e^{i \omega_{n} t}$ growing exponentially. On the other hand if $\omega_{n}$ is purely real the term $e^{i \omega_{n} t}$ remains bounded.

The condition that $\omega_{n}$ is real is equivalent to the following condition on the eigenvalue $\lambda$ in the dispersion relation (4.16):

$$
-1 \leq \cos \left(\omega_{n} \Delta t\right) \leq 1 \quad \Leftrightarrow \quad 0 \leq \lambda \nu^{2} \leq 4 .
$$

Based on the stability condition (6.2) we write the following lemma.

Lemma 6.2. The numerical frequency $\omega_{n}$ is purely real if the following are satisfied

$$
\begin{gathered}
M^{P} \text { is positive definite and } A^{P} \text { is non-negative definite, } \\
\nu \leq\left(\left\|h^{-2} M^{P}\right\|\left\|A^{P}\right\|\right)^{-1 / 2} .
\end{gathered}
$$

Proof. The condition (6.3) is the basis for the Lemma 6.1, which implies the first inequality in $(6.2), 0 \leq \lambda$.

The condition (6.4) implies that

$$
\begin{aligned}
\lambda & \leq\left\|\bar{M}^{P} \bar{A}^{P}\right\|=\left\|h^{-2}\left(S^{*} M^{P} S\right)\left(S^{*} A^{P} S\right)\right\| \leq\left\|S^{*}\right\|^{2}\|S\|^{2}\left\|h^{-2} M^{P}\right\|\left\|A^{P}\right\| \\
& =\left\|S^{*}\right\|^{2}\|S\|^{2}\left\|h^{-2} M^{P}\right\|\left\|A^{P}\right\|=4\left\|h^{-2} M^{P}\right\|\left\|A^{P}\right\| .
\end{aligned}
$$

Here we first used the definitions (4.16) of $\bar{W}^{P}$ and $\bar{A}^{P}$ and that $\|S\|=\left\|S^{*}\right\|=\sqrt{2}$. We can satisfy Equation (6.4) by applying the above estimate on $\lambda$ to the second inequality of of (6.2), $\lambda \nu^{2} \leq 4$ and solving for $\nu$.

Using the above Lemma 6.2 we were able to prove the following three conservative stability bounds.

Theorem 6.3. Nédélec's Discretization satisfies the stability condition when $\nu \in\left[0, \frac{1}{\sqrt{2}}\right]$

Proof. We will begin by showing $A^{P}$ and $M_{N}^{P}$ the local stiffness and mass matrices

$$
A^{P}=\left[\begin{array}{rrrr}
1 & 1 & -1 & -1 \\
1 & 1 & -1 & -1 \\
-1 & -1 & 1 & 1 \\
-1 & -1 & 1 & 1
\end{array}\right], \quad M_{N}^{P}=\frac{h^{2}}{6}\left[\begin{array}{llll}
2 & 0 & 1 & 0 \\
0 & 2 & 0 & 1 \\
1 & 0 & 2 & 0 \\
0 & 1 & 0 & 2
\end{array}\right]
$$


As we know that the local mass and stiffness matrices $M^{P}$ and $A^{P}$ represent the same discrete bilinear form as $\widetilde{M}^{P}$ and $\widetilde{A}^{P}$ in a different basis. Therefore $M^{P}$ is positive definite if an only if $\tilde{M}^{P}$ is positive definite and $A^{P}$ is non-negative definite if and only if $\tilde{A}^{P}$ is non-negative definite. The singular values of $A^{P}$ are 4 and 0 (which has multiplicity 3 ). The singular values of $M^{P}$ are given by $1 / 2$ and $1 / 6$, each with multiplicity 2 . Therefore,

$$
\left\|A^{P}\right\|_{2}=4, \quad\left\|h^{-2} M_{N}^{P}\right\|_{2}=1 / 2, \quad\left(\left\|h^{-2} M_{N}^{P}\right\|\left\|A^{P}\right\|\right)^{-1 / 2}=\frac{1}{\sqrt{2}} .
$$

To produce the final stability condition we apply Lemma 6.2.

Theorem 6.4. The GY-adaptation method satisfies the stability condition for $\nu \in[0, \sqrt{3 / 8}]$

Proof. Note that $A^{P}$ is the same for the GY family as for Nédélec's discretization. Therefore $\left\|A^{P}\right\|=4$. The mass matrix $M_{G Y}^{P}$ is positive definite as $a_{M}>0$. We begin by presenting the local mass matrix:

$$
M_{G Y}^{P}=\frac{h^{2}}{12}\left[\begin{array}{rrrr}
7 & 0 & -1 & 0 \\
0 & 7 & 0 & -1 \\
-1 & 0 & 7 & 0 \\
0 & -1 & 0 & 7
\end{array}\right]
$$

The singular values of $h^{-2} M_{G Y}^{P}$ are $\frac{2}{3}$ and $\frac{1}{2}$ so $\left\|h^{-2} M_{G Y}^{P}\right\|=\frac{2}{3}$. We now calculate

$$
\left(\left\|h^{-2} M_{G Y}^{P}\right\|\left\|A^{P}\right\|\right)^{1 / 2}=\sqrt{\frac{3}{8}}
$$

to produce the final condition on $\nu$.

Theorem 6.5. The $M$-adaptation method satisfies the stability condition for $\nu \in[0, \sqrt{3 / 8}]$.

Proof. The stiffness matrix $A^{P}$ is the same as in the two other methods. For the choice of $\nu^{2} \leq 2$ the matrix $M^{P}$ is positive definite. The local mass matrix $M_{M F D}^{P}$ in the d.o.f. basis is

$$
M_{M F D}^{P}=\frac{h^{2}}{12}\left[\begin{array}{rrrr}
7 & 0 & -1 & 0 \\
0 & 7 & 0 & -1 \\
-1 & 0 & 7 & 0 \\
0 & -1 & 0 & 7
\end{array}\right]-\frac{h^{2} \nu^{2}}{12}\left[\begin{array}{rrrr}
1 & 1 & -1 & -1 \\
1 & 1 & -1 & -1 \\
-1 & -1 & 1 & 1 \\
-1 & -1 & 1 & 1
\end{array}\right]
$$

We used MATHEMATICA to calculate the singular values of $h^{-2} M^{P}$, which are given by $\frac{2}{3}, \frac{1}{2}, \frac{1}{3}\left|2-\nu^{2}\right|$. For the Courant number $\nu$ in the region $(0, \sqrt{2}), \frac{1}{3}\left|2-\nu^{2}\right| \leq \frac{2}{3}$. Therefore, $\left\|h^{-2} M_{M F D}^{P}\right\|=\frac{2}{3}$ for the relevant $\nu$. As this is the same as GY case we reach the same conclusion.

Remark. The mass matrix (6.9) is written in such a way that it is obvious that

$$
h^{-2} M_{M F D}^{P}=h^{-2} M_{G Y}^{P}-\frac{\nu^{2}}{12} A^{P} .
$$


The above may prove useful in understanding the relationship between the two methods and proving the order of accuracy of the MFD scheme.

During our numerical experiments we found that our conservative stability estimates were too conservative as the methods appeared to work outside of the proven stability region. In the subsequent section we produce tight bounds on the eigenvalue $\lambda$ which produce less conservative stability regions.

\subsection{Tight stability bounds}

The norm-based stability estimates are not optimal. We will now produce optimal estimates by working directly with the non-zero eigenvalue, $\lambda$, of the derived matrix product $B=\bar{M}^{P} \bar{A}^{P}$. Let $b_{i j}=[B]_{i j}$. As $\operatorname{det}(B)=0$ then $\lambda=\operatorname{tr}(B)$. The expression for $\lambda$ can be greatly simplified if written in terms of the following two parameters:

$$
\begin{aligned}
& \alpha=\sin ^{2}\left(\frac{k_{1} h}{2}\right), \\
& \beta=\sin ^{2}\left(\frac{k_{2} h}{2}\right) .
\end{aligned}
$$

where $k_{1}=k \cos \theta$ and $k_{2}=k \sin \theta$. As $k_{1} h$ and $k_{2} h$ can take arbitrary, independent values, $\alpha$ and $\beta$ can each take independent values in $[0,1]$. A discretization will be stable at a particular $\nu$ if it satisfies the following two conditions

$$
\min _{(\alpha, \beta) \in[0,1]^{2}} \lambda\left(\alpha, \beta, \nu^{2}\right) \nu^{2} \geq 0 \quad \text { and } \quad \max _{(\alpha, \beta) \in[0,1]^{2}} \lambda\left(\alpha, \beta, \nu^{2}\right) \nu^{2} \leq 4
$$

We can define the stability region of a discretization as $\left[0, \nu_{\text {crit }}\right]$ where $\nu_{\text {crit }}$ is the maximum Courant number which satisfies equation (6.12). The following three sections, we will compute $\nu^{*}$ for each discretization. In Figure 4 we plot the maximum from equation (6.12).

\subsubsection{Nédélec discretization}

We will now compute $\nu^{*}$ for the mass lumped Nédélec discretization. We express $B$ as follows:

$$
\begin{aligned}
b_{11} & =\frac{4}{3} \alpha(3-2 \alpha), \\
b_{12} & =e^{\mathrm{i} \frac{h}{2}\left(k_{1}+k_{2}\right)} \frac{4}{3}(\alpha \beta)^{1 / 2}(3-2 \alpha), \\
b_{21} & =e^{-\mathrm{i} \frac{h}{2}\left(k_{1}+k_{2}\right)} \frac{4}{3}(3-2 \beta), \\
b_{22} & =\frac{4}{3} \beta(3-2 \beta) .
\end{aligned}
$$

As $\operatorname{det} B=0$, the non-zero eigenvalue $\lambda=\operatorname{tr}(B)$ :

$$
\lambda=\frac{4}{3}(\alpha(3-2 \alpha)+\beta(3-2 \beta)) .
$$

Therefore $0 \leq \lambda \leq 3$, we find that $\nu_{\text {crit }}=\frac{2}{\sqrt{3}}$. 


\subsubsection{GY-adaptation}

We proceed as for the Nédélec discretization.

$$
\begin{aligned}
b_{11} & =\frac{4}{3} \alpha(3+\alpha) \\
b_{12} & =e^{\mathrm{i} \frac{h}{2}\left(k_{1}+k_{2}\right)} \frac{4}{3}(\alpha \beta)^{1 / 2}(3+\alpha), \\
b_{21} & =e^{-\mathrm{i} \frac{h}{2}\left(k_{1}+k_{2}\right)} \frac{4}{3}(\alpha \beta)^{1 / 2}(3+\beta), \\
b_{22} & =\frac{4}{3} \beta(3+\beta) .
\end{aligned}
$$

Similar to the Nédélec discretization, $\operatorname{det}(B)=0$. We can then use $\operatorname{tr}(B)$ to calculate that the non-zero eigenvalue

$$
\lambda=\frac{4}{3}(\alpha(3+\alpha)+\beta(3+\beta)) .
$$

Therefore $0 \leq \lambda \leq \frac{32}{3}$, we find that $\nu_{\text {crit }}=\sqrt{\frac{3}{8}}$.

\subsubsection{M-adaptation}

We can then express $B$ as follows:

$$
\begin{aligned}
& b_{11}=-\frac{4}{3} \alpha \beta \nu^{2}+\frac{\alpha}{3}\left(6+2\left(1-\nu^{2}\right) \alpha\right), \\
& b_{12}=e^{\mathrm{i} \frac{h}{2}\left(k_{1}+k_{2}\right)} \frac{2}{3}(\alpha \beta)^{1 / 2}\left(6+2\left(1-\nu^{2}\right) \alpha-2 \nu^{2} \beta\right), \\
& b_{21}=e^{-i \frac{h}{2}\left(k_{1}+k_{2}\right)} \frac{2}{3}(\alpha \beta)^{1 / 2}\left(6+2\left(1-\nu^{2}\right) \beta-2 \nu^{2} \alpha\right), \\
& b_{22}=-\frac{4}{3} \alpha \beta \nu^{2}+\frac{\beta}{3}\left(6+2\left(1-\nu^{2}\right) \beta\right) .
\end{aligned}
$$

We can compute the eigenvalue using the trace and note that

$$
\lambda(\alpha, \beta, \nu)=\frac{1}{3}\left(-8 \alpha \beta \nu^{2}+\alpha\left(6+2\left(1-\nu^{2}\right) \alpha\right)+\beta\left(6+2\left(1-\nu^{2}\right) \beta\right)\right) .
$$

The above computation allows us to find that $\nu_{\text {crit }}=\frac{1}{\sqrt{2}}$. See Figure 4 for numerical evidence.

\section{Numerical Simulations}

In the following few sections we present a number of numerical experiments designed to illustrate the convergence properties for the numerical dispersion and numerical anisotropy derived analytically in Section 5.

\subsection{Phase error convergence}

In order to present a rate convergence for the numerical speed we must first adapt our analysis to computable problem. As the domain of our analysis is $\mathbb{R}^{2}$ we will use periodic 


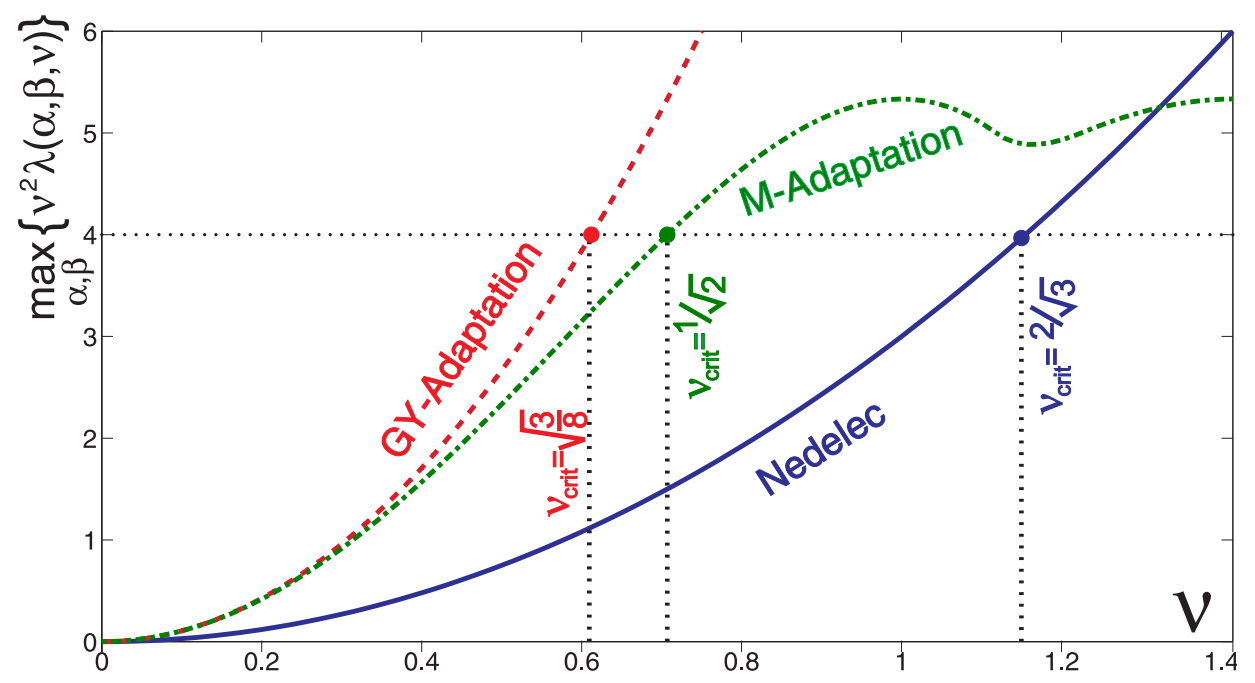

Figure 4: Numerically computed values of the maximum of $\nu^{2} \lambda(\alpha, \beta, \nu)$ where $(\alpha, \beta) \in[0,1]^{2}$. In this case $\lambda(\alpha, \beta, \nu)$ is computed at each $\nu$ on a uniform mesh of 10,000 grid points in $\alpha, \beta$ space and the maximum is selected from these samples.
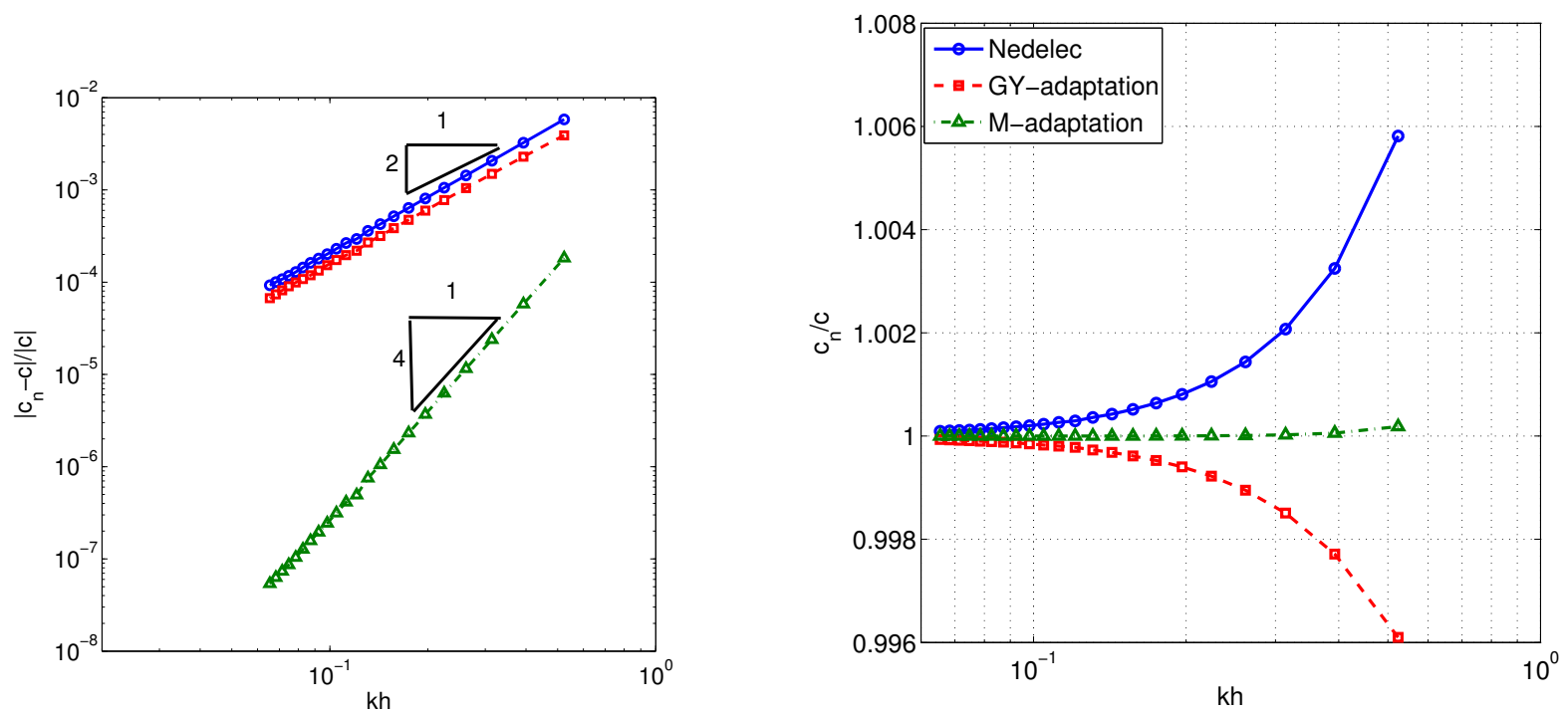

Figure 5: The relative errors in the numerical wave speed $c_{n}$ for planar waves aligned with the mesh $(\theta=0)$ for various resolutions of the wave characterized by the number of points per wavelength between in the range between 12 and 96. The Courant number $\nu=\frac{2}{\sqrt{3}}$ for the Nédélec method, $\nu=\frac{1}{\sqrt{2}}$ for the M-adaptation method, and $\nu=\sqrt{\frac{3}{8}}$ for the GY-adaptation method.

boundary conditions and solve the following initial value problem:

$$
\begin{cases}\frac{\partial^{2}}{\partial t^{2}} \mathbf{E}+c^{2} \text { curl curl } \mathbf{E}=0 & \text { on }\left[-\frac{1}{2}, \frac{1}{2}\right] \times\left[-\frac{1}{2}, \frac{1}{2}\right], \\ \mathbf{E}\left(-\frac{1}{2}, y\right)=\mathbf{E}\left(\frac{1}{2}, y\right), \mathbf{E}\left(x,-\frac{1}{2}\right)=\mathbf{E}\left(x, \frac{1}{2}\right), & \\ \frac{\partial}{\partial t} \mathbf{E}\left(-\frac{1}{2}, y\right)=\mathbf{E}\left(\frac{1}{2}, y\right) \dot{\mathbf{E}}\left(x,-\frac{1}{2}\right)=\dot{\mathbf{E}}\left(x, \frac{1}{2}\right) . & \end{cases}
$$


Further, after we discretizing the system we will use the initial conditions corresponding to the classical solution of the plane wave:

$$
\begin{aligned}
\mathbf{U}^{0} & =\mathbf{k}^{\perp} \exp (\mathrm{ik} \cdot \mathbf{x}), \\
\mathbf{U}^{-1} & =\mathbf{k}^{\perp} \exp (\mathrm{ik} \cdot \mathbf{x}+\mathrm{i} c k \Delta t) .
\end{aligned}
$$

In order to produce the numerical speed from calculations of the degrees of freedom we track the location of a local maximum of the degrees of freedom lying along the $x$ and $y$ axes. The location and value of this maximum and its nearest neighbors is quadratically interpolated to estimate the location of the front. We then use a least squares linear regression of times and location of the front to produce a numerical velocity.

From this a speed in the $x$ and $y$ direction is computed and the total speed is determined to be the Euclidean norm of these values. See Figure 5 for results. For this experiment we recovered $\mathcal{O}(k h)^{2}$ convergence in relative phase error, $\frac{\left|c-c_{n}\right|}{|c|}$, for the Nédélec and GYadaptation methods and $\mathcal{O}(k h)^{4}$ relative phase error for the M-adaptation. This experiment is consistent with our theoretical results above.

\subsection{Numerical anisotropy}

In this section we wish to confirm the convergence rates for the numerical anisotropy analytically shown in Section 5 on a set of numerical experiment. Unlike the case of waves oriented with the mesh where periodic conditions are simple to define, for waves not aligned with the mesh extra care must be taken to enforce continuity across the periodic edges.

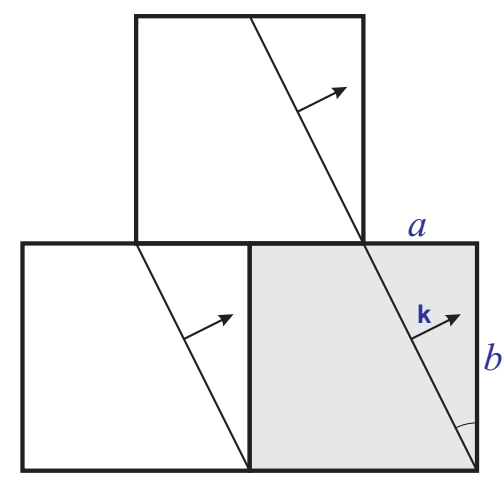

Figure 6: Proper selection of $\theta$ enforces continuity over the top and bottom boundaries of the domain, while $k$ determines continuity across left and right boundaries.

By choosing angles of propagation $\theta$ such that $\tan \theta=\frac{a}{b}, a, b \in \mathbb{Z}$ and choosing wave numbers $k=2 \pi b \sec \theta$ the necessary continuity is guaranteed. This produces a plane wave which is continuous over the edges on the interval $[-1 / 2,1 / 2] \times[-1 / 2,1 / 2]$. The number of points per wavelength can then be increased to any tolerance by taking mesh size $h$ sufficiently small. See Figure 6 for an illustration.

Due to various symmetry considerations (mesh rotations and reflections), we will only consider waves forming angles $\theta \in\left[0, \frac{\pi}{4}\right]$ with the mesh. Figure 7 shows the relative dispersion errors at for waves with 12 and 24 points per wavelength. The displayed results are consistent with the analytically results of Section 5 . We note that for the angle $\theta=\pi / 4$ (i.e., 


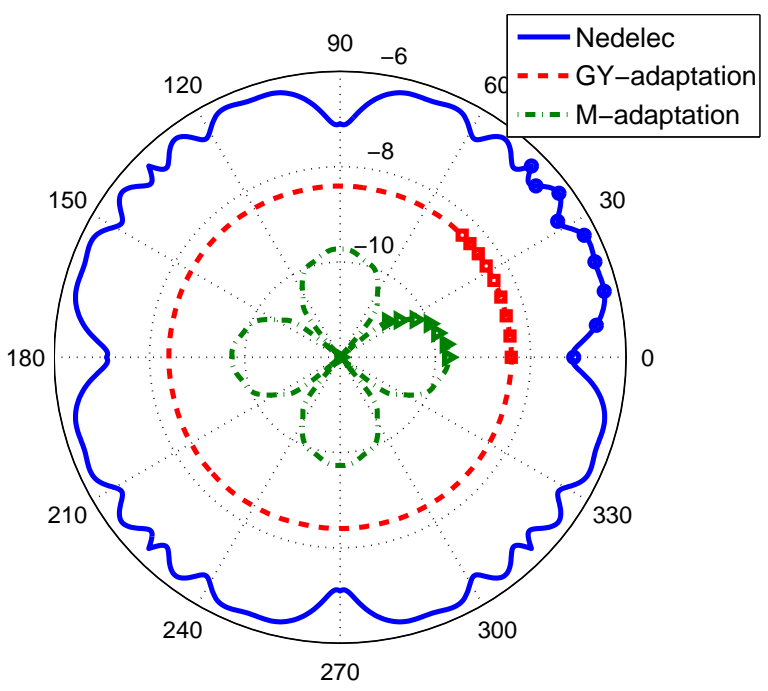

12 points per wavelength

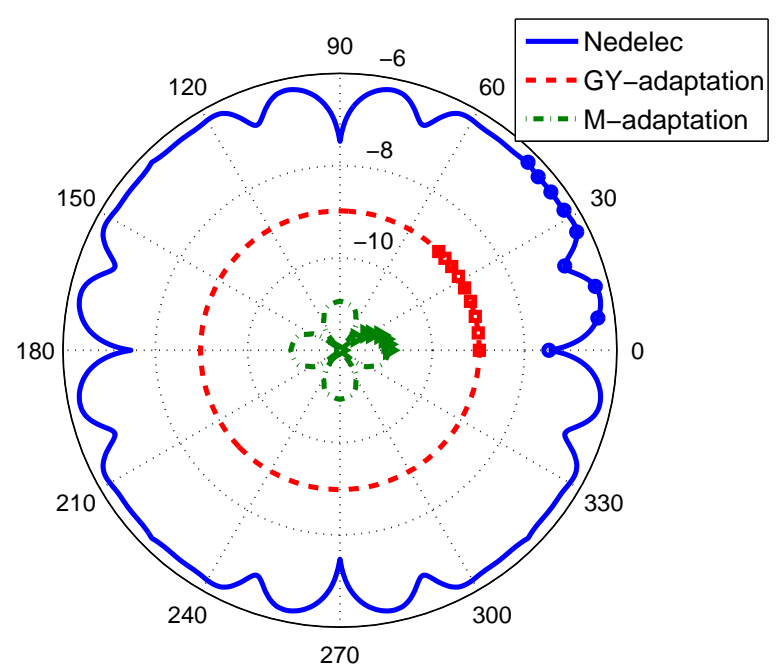

24 points per wavelength

Figure 7: Log-scale polar plots of relative dispersion error computed on the first half of the first quadrant and symmetrically extended to all directions of wave propagation, i.e., actual computations were done for planar wave angles $\theta \in[0, \pi / 4]$. The data points are marked with markers and the smooth curves are constructed using cubic splines interpolation. In all experiments the largest stable time step was used.

wave moving diagonally relative to the mesh) the M-adaptation displays additional superconvergence. In particular, even for waves with twelve points per wavelength M-adaptation leads to a numerical dispersion which is below machine precision.

\subsection{Demonstration of Convergence in $\mathbf{L}^{2}$ and Energy Norms}

In this section we demonstrate the convergence of the numerical solution of the electric vector wave equation obtained using the Nédélec, GY and MFD schemes in the $\mathbf{L}^{2}$ and the energy norms, where the energy norm for the electric field is defined as

$$
\|\mathbf{E}\|_{\mathcal{E}}:=\sqrt{\int_{\Omega}|\mathbf{E}|^{2}+c^{2} \int_{\Omega}|\operatorname{curl} \mathbf{E}|^{2}} .
$$

Consider the function

$$
\mathbf{E}^{i, j}(\mathbf{x}, t)=\cos (\omega \pi t)\left[\begin{array}{r}
\frac{j}{\omega \epsilon \sqrt{\mu}} \cos (i \pi x) \sin (j \pi y) \\
-\frac{i}{\omega \epsilon \sqrt{\mu}} \sin (i \pi x) \cos (j \pi y)
\end{array}\right] \quad \omega=\sqrt{\frac{i^{2}+j^{2}}{\epsilon \mu}} \text { for } i, j \in \mathbb{Z} .
$$

For simplicity we choose $\epsilon=\mu=c=1$. For every pair of integers $i$ and $j$ the function $\mathbf{E}^{i, j}$ 
is an exact solution of the following initial boundary value problem:

$$
\begin{array}{lr}
\frac{\partial^{2}}{\partial t^{2}} \mathbf{E}^{i, j}+c^{2} \mathbf{c u r l} \text { curl } \mathbf{E}^{i, j}=0 & \text { in } \Omega=[0,1]^{2}, t>0, \\
\mathbf{E}^{i, j} \times \mathbf{n}=0 & \text { on } \partial \Omega, t>0, \\
\mathbf{E}^{i, j}(\mathbf{x}, 0)=\mathbf{E}^{i, j}(\mathbf{x}, 0), & \\
\frac{\partial}{\partial t} \mathbf{E}^{i, j}(\mathbf{x}, 0)=\mathbf{0} . &
\end{array}
$$

Table 1: $\mathbf{L}^{2}$ errors and rates for $\mathbf{E}^{1,1}$.

\begin{tabular}{|c|c||c|c||c|c||c|c|}
\hline$h$ & $N_{\text {p.p.w. }}$ & $\log _{2} e_{\mathbf{L}^{2}}^{N}$ & N rate & $\log _{2} e_{\mathbf{L}^{2}}^{G}$ & GY rate & $\log _{2} e_{\mathbf{L}^{2}}^{M}$ & M rate \\
\hline \hline $2^{-5}$ & 142 & -6.5223 & & -8.1234 & & -18.6520 & \\
$2^{-6}$ & 284 & -8.5324 & 2.0101 & -10.1213 & 1.9979 & -23.6471 & 4.9952 \\
$2^{-7}$ & 568 & -10.5355 & 2.0031 & -12.1214 & 2.0001 & -28.6463 & 4.9990 \\
$2^{-8}$ & 1,137 & -12.5366 & 2.0011 & -14.1218 & 2.0003 & -33.6309 & 4.9847 \\
\hline
\end{tabular}

Table 2: Energy errors and rates for $\mathbf{E}^{1,1}$.

\begin{tabular}{|c|c||c|c||c|c||c|c|}
\hline$h$ & $N_{\text {p.p.w. }}$ & $\log _{2} e_{\mathcal{E}}^{N}$ & N rate & $\log _{2} e_{\mathcal{E}}^{G}$ & GY rate & $\log _{2} e_{\mathcal{E}}^{M}$ & M rate \\
\hline \hline $2^{-5}$ & 142 & -4.3368 & & -5.9363 & & -17.0760 & \\
$2^{-6}$ & 284 & -6.3455 & 2.0088 & -7.9344 & 1.9979 & -21.1318 & 4.0557 \\
$2^{-7}$ & 568 & -8.3483 & 2.0028 & -9.9342 & 2.0001 & -25.1397 & 4.0079 \\
$2^{-8}$ & 1,137 & -10.3495 & 2.0010 & -11.9347 & 2.0003 & -29.1230 & 3.9832 \\
\hline
\end{tabular}

Table 3: $\mathbf{L}^{2}$ errors and rates for $\mathbf{E}^{4,4}$.

\begin{tabular}{|c|c||c|c||c|c||c|c|}
\hline$h$ & $N_{\text {p.p.w. }}$ & $\log _{2} e_{\mathbf{L}^{2}}^{N}$ & N rate & $\log _{2} e_{\mathbf{L}^{2}}^{G}$ & GY rate & $\log _{2} e_{\mathbf{L}^{2}}^{M}$ & M rate \\
\hline \hline $2^{-5}$ & 35 & -0.5717 & & -2.3715 & & -8.7529 & \\
$2^{-6}$ & 71 & -2.3941 & 1.8223 & -4.1348 & 1.7634 & -13.6724 & 4.9192 \\
$2^{-7}$ & 142 & -4.4597 & 2.0656 & -6.0904 & 1.9556 & -18.6523 & 4.9800 \\
$2^{-8}$ & 294 & -6.4837 & 2.0239 & -8.0806 & 1.9900 & -23.6471 & 4.9949 \\
\hline
\end{tabular}

Table 4: Energy errors and rates for $\mathbf{E}^{4,4}$.

\begin{tabular}{|c|c||c|c||c|c||c|c|}
\hline$h$ & $N_{\text {p.p.w. }}$ & $\log _{2} e_{\mathcal{E}}^{N}$ & $\mathrm{~N}$ rate & $\log _{2} e_{\mathcal{E}}^{G}$ & GY rate & $\log _{2} e_{\mathcal{E}}^{M}$ & M rate \\
\hline $2^{-5}$ & 35 & 3.5541 & & 1.7822 & & -7.0289 & \\
$2^{-6}$ & 71 & 1.7526 & 1.8015 & 0.0189 & 1.7632 & -11.1454 & 4.1166 \\
$2^{-7}$ & 142 & -0.3076 & 2.0604 & -1.9367 & 1.9556 & -15.1453 & 3.9999 \\
$2^{-8}$ & 294 & -2.3303 & 2.0226 & -3.9265 & 1.9900 & -19.1061 & 3.9941 \\
\hline
\end{tabular}

For this problem we will refer to the numerically computed solution as $\mathbf{E}_{h}^{*}$, where $*=$ $N, G, M$ indicates the numerical method used to obtain the solution. Here $*=N$ stands for Nédélec, $*=G$ stands for GY-adaptation, and $*=M$ for M-adaptation. For each method, we ran our simulations at the maximum stable time-step, c.f. Figure 4, and for each method we computed a fixed number of time steps which is an integer multiple of the Courant number for the respective scheme. We computed initial conditions exactly and used 
Table 5: $\mathbf{L}^{2}$ errors and rates for $\mathbf{E}^{1,4}$.

\begin{tabular}{|c|c||c|c||c|c||c|c|}
\hline$h$ & $N_{\text {p.p.w. }}$ & $\log _{2} e_{\mathbf{L}^{2}}^{N}$ & N rate & $\log _{2} e_{\mathbf{L}^{2}}^{G}$ & GY rate & $\log _{2} e_{\mathbf{L}^{2}}^{M}$ & M rate \\
\hline \hline $2^{-6}$ & 98 & -3.4588 & & -5.7736 & & -8.3872 & \\
$2^{-7}$ & 195 & -5.2503 & 1.7915 & -7.4233 & 2.0212 & -11.0952 & 2.7079 \\
$2^{-8}$ & 390 & -7.2069 & 1.9564 & -9.8013 & 2.0065 & -13.6458 & 2.5507 \\
$2^{-9}$ & 780 & -9.1964 & 1.9897 & -11.8035 & 2.0021 & -16.0276 & 2.3818 \\
\hline
\end{tabular}

Table 6: Energy errors and rates for $\mathbf{E}^{1,4}$.

\begin{tabular}{|c|c||c|c||c|c||c|c|}
\hline$h$ & $N_{\text {p.p.w. }}$ & $\log _{2} e_{\mathcal{E}}^{N}$ & N rate & $\log _{2} e_{\mathcal{E}}^{G}$ & GY rate & $\log _{2} e_{\mathcal{E}}^{M}$ & M rate \\
\hline \hline $2^{-6}$ & 98 & 0.2335 & & -2.0858 & & -8.3228 & \\
$2^{-7}$ & 195 & -1.3512 & 1.7863 & -4.1003 & 2.0143 & -11.0843 & 2.7615 \\
$2^{-8}$ & 390 & -3.5080 & 1.9551 & -6.1044 & 2.0041 & -13.6645 & 2.5603 \\
$2^{-9}$ & 780 & -5.4971 & 1.9894 & -8.1055 & 2.0012 & -16.0276 & 2.3832 \\
\hline
\end{tabular}

Table 7: $\mathbf{L}^{2}$ errors and rates for $\mathbf{E}^{2,8}$.

\begin{tabular}{|c|c||c|c||c|c||c|c|}
\hline$h$ & $N_{\text {p.p.w. }}$ & $\log _{2} e_{\mathbf{L}^{2}}^{N}$ & $\mathrm{~N}$ rate & $\log _{2} e_{\mathbf{L}^{2}}^{G}$ & GY rate & $\log _{2} e_{\mathbf{L}^{2}}^{M}$ & M rate \\
\hline \hline $2^{-6}$ & 48 & -1.7981 & & -2.3848 & & -4.7301 & \\
$2^{-7}$ & 98 & -1.7812 & 0.0730 & -4.3139 & 1.9292 & -7.7039 & 2.9737 \\
$2^{-8}$ & 195 & -3.6930 & 1.9120 & -6.3024 & 1.9887 & -10.6574 & 2.9535 \\
$2^{-9}$ & 390 & -5.6878 & 1.9948 & -8.3002 & 1.9978 & -13.5697 & 2.9124 \\
\hline
\end{tabular}

Table 8: Energy errors and rates for $\mathbf{E}^{2,8}$.

\begin{tabular}{|c|c||c|c||c|c||c|c|}
\hline$h$ & $N_{\text {p.p.w. }}$ & $\log _{2} e_{\mathcal{E}}^{N}$ & $\mathrm{~N}$ rate & $\log _{2} e_{\mathcal{E}}^{G}$ & GY rate & $\log _{2} e_{\mathcal{E}}^{M}$ & M rate \\
\hline \hline $2^{-6}$ & 48 & 2.9545 & & 2.2941 & & -3.2794 & \\
$2^{-7}$ & 98 & 2.9083 & 0.0461 & 0.3780 & 1.9158 & -7.0056 & 3.7260 \\
$2^{-8}$ & 195 & 1.0016 & 1.9070 & -1.6075 & 1.9856 & -10.4205 & 3.4147 \\
$2^{-9}$ & 390 & -0.9919 & 1.9935 & -3.6053 & 1.9970 & -13.5096 & 3.0895 \\
\hline
\end{tabular}

Mass and Stiffness matrices to compute norms. To compute the $\mathbf{L}^{2}$-error using the mass matrix, one must solve a linear system as only the inverse mass matrix $W$ is available to us. We use conjugate gradient methods to solve the system set to the tolerance of $10^{-14}$ in this experiment.

Our results suggest that the Nédélec and GY-adaptation Methods are second order in both $\mathbf{L}^{2}$ and Energy norms. We observed super quadratic convergence for the M-adaptation method, and for the case of the $\mathbf{E}^{4,4}$ mode we observed a fifth order convergence in mesh resolution. For the $\mathbf{E}^{1,4}$ and $\mathbf{E}^{2,8}$ modes we observed convergence rates much closer to $\mathcal{O}\left(h^{2}\right)$. This suggests that for general initial conditions the MFD method is second order in $\mathbf{L}^{2}$ and Energy norms. We believe that the super-convergence observed for $\mathbf{E}^{j, j}$ modes coincides with super-convergence of numerical dispersion as $\mathbf{E}^{i, j}$ is a linear combination of the imaginary parts of four plane waves where $\mathbf{k}=(i, j),(-i, j),(i,-j)$, and $(-i,-j)$. That is $\mathbf{E}^{j, j}, j \in \mathbb{N}$ is a linear combination of four plane waves propagating at $45^{\circ}$ angle to the mesh axis, exactly the angle at which we observed super-convergence in numerical dispersion. For each of the 

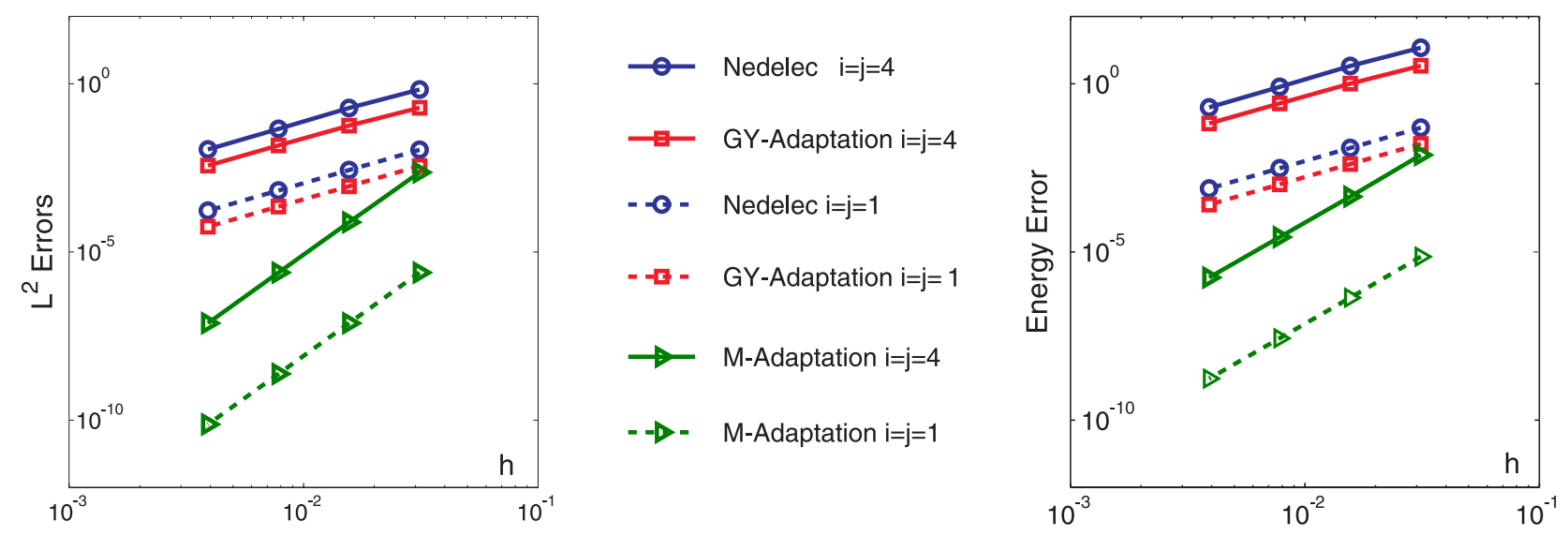

Figure 8: The $\mathbf{L}^{2}$ (left) and Energy (right) errors for $\mathbf{E}^{1,1}$ and $\mathbf{E}^{4,4}$ solutions.
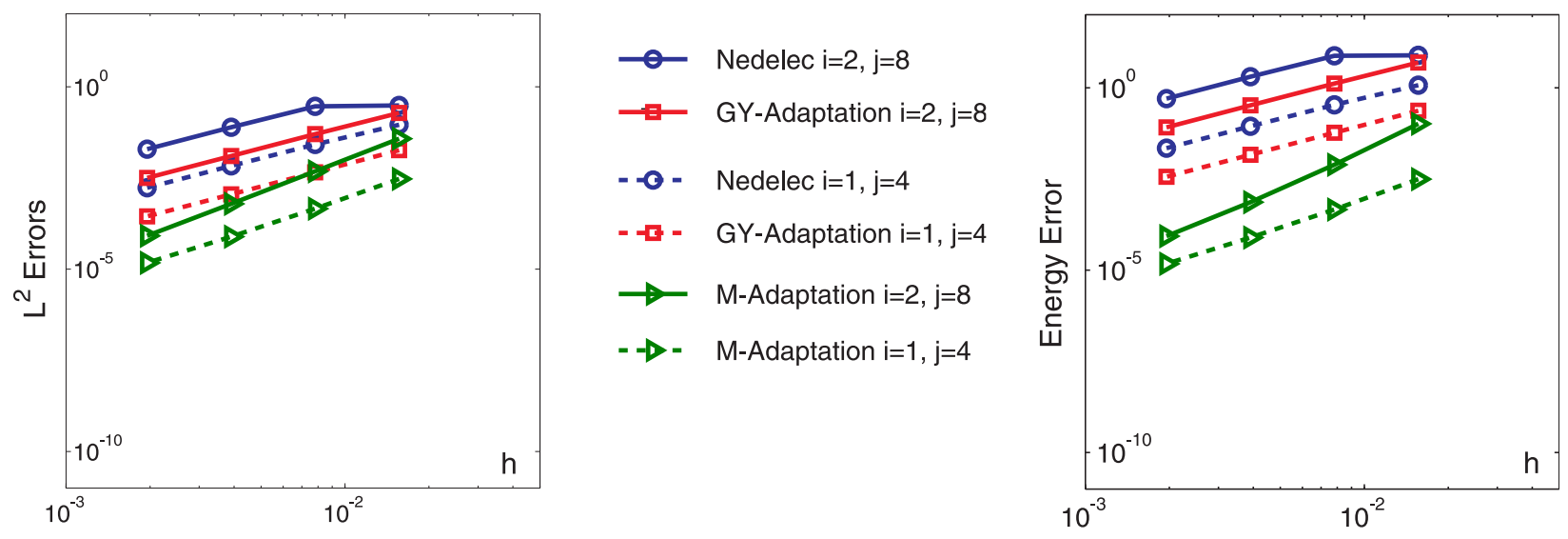

Figure 9: The $\mathbf{L}^{2}$ (left) and Energy (right) errors for $\mathbf{E}^{1,4}$ and $\mathbf{E}^{2,8}$ solutions.

methods $*=N, G, M$ we define the absolute error in the norm $\mathbf{V}$ as

$$
\mathrm{e}_{\mathbf{V}}^{*}:=\left\|\mathbf{E}_{h}^{*}-\mathbf{E}\right\|_{\mathbf{V}} \text {, where } \mathbf{V} \text { is the norm and } * \text { is the method. }
$$

In Tables 1-8 and Figures 8 and 9 we collect all the results of this experiment. For each of the simulations the mesh resolution of the wave is computed as a number of points per wavelength $\left(N_{\text {p.p.w. }}\right)$ by rounding the quantity $\frac{2 \pi c}{\omega h}$ to the nearest integer. We believe that suboptimal results displayed for $\mathbf{E}^{2,8}$ for the Nédélec method may be due to relatively low mesh resolution.

\section{Conclusions}

In this paper, we have constructed and analyzed three different methods for the spatial discretization of the two dimensional electric vector wave equation in the time domain. All three methods use the explicit Leapfrog time integration for their temporal evolution in order to produce efficient time domain methods for long time integration. An analysis based

on stability and dispersion is performed for all three approaches that indicates the different 
advantages and disadvantages of the three methods.

All three approaches use a nonstandard lumping method on the mass matrix. In the case of the lowest order Nédélec edge finite elements, the local mass and stiffness matrices are computed exactly and the lumping is applied in the global assembly. In the GY-adaptation method the local mass and stiffness matrices are computed using a four-point quadrature rule which produces a one parameter $\left(a_{M}\right)$ family of mass matrix discretizations. The stiffness matrix turns out to be parameter independent and identical to that in the Nédélec method. The Nédélec method is a special member of the GY family of discretizations. The mimetic M-adaptation approach is a generalization of the GY-adaptation technique, including the Nédélec method and the GY family as special cases, and produces a three parameter $\left(m_{1}, m_{2}, m_{3}\right)$ based family of discretizations. Using dispersion analysis of the parametric families we obtain the optimal members of the GY and M-adaptation discretizations that have minimum dispersion error. We show that the mass lumping technique used in this paper produces a Nédélec method which has second order phase error and second order numerical anisotropy. The optimal member of the GY family has second order numerical phase error and fourth order numerical anisotropy, while the optimal member of the mimetic family has fourth order numerical phase error and fourth order numerical anisotropy in its stable region.

There are other considerations that need to be accounted for in an overall comparison of the three methods ( Nédélec, GY-adaptation, M-adaptation). We show that the local mass inverse is $2 \times 2$ block diagonal if $m_{1}=m_{3}$ and $m_{2}=0$, as is the case of the Nédélec and GY-adaptation schemes. When $m_{1} \neq m_{3}$ the local mass inverse is a $4 \times 4$ dense matrix. Thus density of the global lumped mass matrix $W_{G}$ in the case of the M-adaptation scheme is roughly double that of the GY and Nédélec discretizations.

We performed a von Neumann analysis and found that the Nédélec method is stable for $\nu \in\left[0, \frac{2}{\sqrt{3}}\right]$, GY-adaptation is stable for $\nu \in\left[0, \sqrt{\frac{3}{8}}\right]$, and M-adaptation is stable for $\nu \in\left[0, \frac{1}{\sqrt{2}}\right]$. It should be noted that the Yee Scheme is stable for $\nu \in\left[0, \frac{1}{\sqrt{2}}\right]$. Therefore the Nédélec method is more stable than the Yee Scheme, M-adaptation is as stable as the Yee scheme, and GY-adaptation is less stable than the Yee Scheme.

In terms of other error metrics, our experiments suggest that the Nédélec and GYadaptation methods have order $\mathcal{O}\left(h^{2}\right) \mathbf{L}^{2}$ and Energy Error. For certain initial conditions we observed that the M-adaptation method has super quadratic convergence rates in both these metrics. Given that some of our rates for M-adaptation were fairly close to two we suspect that there may be initial conditions for which the method exhibits only quadratic convergence rates.

An interesting property of the optimal GY-adaptation method is that, unlike methods such as the Yee scheme [31], equations where the lowest dispersion error is attained when the largest stable time step is taken, dispersion error decreases as the time step decreases. Thus small time steps will reduce numerical dispersion error. Further the lack of anisotropy at the second order may make the optimal GY-adaptation method desirable over the Nédélec approach in situations when a radially symmetric pulse is being simulated.

The M-adaptation method's fourth order dispersion error comes at a slight cost increase. However, as demonstrated experimentally in our simulations, the superconvergence along the diagonal in the M-adaptation method could be of great use in the simulation of plane waves for scattering problems. In such a case the plane wave could be propagated along the diagonal 
with machine-tolerance dispersion error at a mere twelve points per wavelength. Thus, for problems where matching wave speeds is critical, and especially where high resolution is computationally infeasible, the M-adaptation method appears to be most desirable.

\section{Acknowledgments}

The authors would like to thank Dr. Konstantin Lipnikov of the Los Alamos National Laboratory for his advice and support during the writing of this paper.

V. Gyrya's work was carried out under the auspices of the National Nuclear Security Administration of the U.S. Department of Energy at Los Alamos National Laboratory under Contract No. DE-AC52-06NA25396 and the DOE Office of Science Advanced Scientific Computing Research (ASCR) Program in Applied Mathematics Research.

V. A. Bokil's and D. McGregor's work was partially supported by the National Science Foundation Grant Number \#0811223. D. McGregor received additional support from the T5 group of the Los Alamos National Laboratory.

Lastly, the authors would like to thank the referees for helpful suggestions.

\section{References}

[1] M. Ainsworth. Dispersive properties of high-order Nédélec/edge element approximation of the time-harmonic maxwell equations. Philos. T. R. Soc. A, 362(1816):471-491, 2004.

[2] R. A. Albanese, J. W. Penn, and R. L. Medina. Short-rise-time microwave pulse propagation through dispersive biological media. J. Opt. Soc. of Am. A, 6:1441-1446, 1989.

[3] V. A. Bokil and N. L. Gibson. Analysis of spatial high-order finite difference methods for Maxwell's equations in dispersive Media. IMA J. Numer. Anal., 32(3):926-956, 2010.

[4] F. Brezzi, A. Buffa, and K. Lipnikov. Mimetic finite differences for elliptic problems. M2AN Math. Model. Numer. Anal., 43(2):277-295, 2009.

[5] G. C. Cohen. Higher-Order Numerical Methods for Transient Wave Equations. Springer, 2002.

[6] J. D. De Basabe and M. K. Sen. Stability of the high-order finite elements for acoustic or elastic wave propagation with high-order time stepping. Geophysical Journal International, 181(1):577-590, 2010.

[7] A. Fisher, R. N. Rieben, G. H. Rodrigue, and D. A. White. A generalized mass lumping technique for vector finite-element solutions of the time-dependent maxwell equations. IEEE Trans. on Antennas Propag., 53(9), 2005.

[8] M. Guddati and B. Yue. Modified integration rules for reducing dispersion error in finite element methods. Comput. Methods Appl. Mech. Engrg., 193:275-287, 2004.

[9] V. Gyrya and K. Lipnikov. M-Adaptation method for acoustic wave equation on square meshes. J. Comput. Acoust., 20(4):1250022-1 - 1250022-23, 2012. 
[10] V. Gyrya and K. Lipnikov. M-adaptation method for acoustic wave equation on rectangular meshes. In A. Cangiani, editor, Proceedings of ENUMATH 2011, the 9th European conference on numerical mathematics and advanced applications, Leicester, UK, September 5-9, 2011, Numerical mathematics and advanced applications 2011, pages 429-439. Springer, 2013.

[11] V. Gyrya, K. Lipnikov, G. Manzini, and D. Svyatskiy. M-adaptation in the mimetic finite difference method. uner review, 2013.

[12] B. Jiang, J. Wu, and L. Povinelli. The origin of spurious solutions in computational electromagnetics. J. Comp. Phys., 125(1):104-123, 1996.

[13] C. Jo, C. Shin, and J. H. Suh. An optimal 9-point, finite difference, frequency-space, 2-D scalar wave extrapolator. Geophysics, 61:529-537, 1996.

[14] S. M. R. Kabir, B. M. Rahman, A. Agrawal, and K. T. V. Grattan. Elimination of numerical dispersion from electromagnetic time domain analysis by using resource efficient finite element technique. Pr. Electromagn. Res., 137:487-512, 2013.

[15] S. Krenk. Dispersion-corrected explicit integration of the wave equation. Comput. Methods Appl. Mech. Engrg., 191:975-987, 2001.

[16] J. Lee, D. K. Sun, and Z. J. Cendes. Tangential vector finite elements for electromagnetic field computation. IEEE Trans. Magn., 27(5):4032-4035, 1991.

[17] K. Lipnikov, G. Manzini, and M. Shashkov. Mimetic finite difference method. J. Comp. Phys., 257:1163-1227, 2014.

[18] P. Monk. A Comparison of Three Mixed Methods for the Time-Dependent Maxwell's Equations. SIAM Sci. Stat. Comput., 13:1097-1122, 1992.

[19] P. Monk. An Analysis of Nédélec's method for the Spatial Discretization of Maxwell's Equations. J. Comput. Appl. Math., 47(1):101-121, 1993.

[20] P. Monk. Finite element methods for Maxwell's equations. Oxford University Press, 2003.

[21] R. Mullen and T. Belytschko. Dispersion analysis of Finite Element semidiscretizations of the two-dimensional wave equation. Int. J. Numer. Meth. Eng., 18:11-29, 1982.

[22] G. Mur. The finite-element modeling of three-dimensional electromagnetic fields using edge and nodal elements. IEEE Trans. Antennas Propag., 41(7):948-953, 1993.

[23] G. Mur. Edge elements, their advantages and their disadvantages. IEEE Trans. Magn., 30(5):3552-3557, 1994.

[24] G. Mur and I. E. Lager. On the causes of spurious solutions in electromagnetics. Electromagnetics, 22(4):357-367, 2002.

[25] J. C. Nédélec. Mixed finite elements in $\mathbb{R}^{3}$. Numer. Math., 35(3):315-341, 1980. 
[26] J. C. Nédélec. A new family of mixed finite elements in $\mathbb{R}^{3}$. Numer. Math, 50:57-81, 1986.

[27] A. Sescu, R. Hixon, and A. A. Afjeh. Multidimensional optimization of finite difference schemes for Computational Aeroacoustics. J. Comput. Phys., pages 4563-4588, 2008.

[28] W. S. Smith, A. Razmadze, X. Shao, and J. L. Drewniak. A hierarchy of explicitly low-dispersion FDTD methods for electrically large problems. IEEE Trans.Antennas and Propag., 60(12):5787-5800, 2012.

[29] I. Stekl and R. G. Pratt. Accurate viscoelastic modeling by frequency-domain finite difference using rotated operators. Geophysics, 63(5):1779-1794, 1998.

[30] D. Sun, J. Manges, X. Yuan, and Z. Cendes. Spurious modes in finite-element methods. IEEE Antennas Propag Magazine, 37(5):12-24, 1995.

[31] A. Taflove and S. C. Hagness. Computational Electrodynamics: The Finite-Difference Time-Domain method. Artech House, Norwood, MA, 3rd edition, 2005.

[32] D. A. White. Orthogonal vector basis functions for time domain finite element solution of the vector wave equation [em field analysis]. IEEE Trans. Magn., 35(3):1458-1461, 1999.

[33] K. Yee. Numerical solution of initial boundary value problems involving maxwell's equations in isotropic media. IEEE Trans. Antennas Propag., 14(3):302-307, 1966.

[34] B. Yue and M. N. Guddati. Dispersion-reducing finite elements for transient acoustics. J. Acoust. Soc. America, 118:2132, 2005. 